\title{
Direct Policy Search for Robust Multi-Objective Management of Deeply Uncertain Socio-Ecological Tipping Points
}

\author{
Julianne D. Quinn ${ }^{\mathrm{a}, *}$, Patrick M. Reed ${ }^{\mathrm{a}}$, Klaus Keller ${ }^{\mathrm{b}, \mathrm{c}, \mathrm{d}}$ \\ ${ }^{a}$ School of Civil and Environmental Engineering, Cornell University, Ithaca, NY, USA. \\ Email: jdq8@cornell.edu, patrick.reed@cornell.edu \\ ${ }^{b}$ Department of Geosciences, The Pennsylvania State University, University Park, PA, \\ USA.Email:klaus@psu.edu \\ ${ }^{c}$ Department of Engineering and Public Policy, Carnegie Mellon University, Pittsburgh, \\ $P A, U S A$. \\ ${ }^{d}$ Earth and Environmental Systems Institute, The Pennsylvania State University, \\ University Park, PA, USA.
}

\begin{abstract}
Managing socio-ecological systems is a challenge wrought by competing societal objectives, deep uncertainties, and potentially irreversible tipping points. A classic, didactic example is the shallow lake problem in which a hypothetical town situated on a lake must develop pollution control strategies to maximize its economic benefits while minimizing the probability of the lake crossing a critical phosphorus (P) threshold, above which it irreversibly transitions into a eutrophic state. Here, we explore the use of direct policy search (DPS) to design robust pollution control rules for the town that account for deeply uncertain system characteristics and conflicting objectives. The closed loop control formulation of DPS improves the quality and robustness of key management tradeoffs, while dramatically reducing the computational complexity of solving the multi-objective pollution control problem relative to open loop control strategies. These insights suggest DPS is a promising tool for managing socioecological systems with deeply uncertain tipping points.
\end{abstract}

Keywords: Socio-ecological management, multi-objective decision making,

${ }^{*}$ Corresponding author. Email: jdq8@cornell.edu, Telephone: 1-603-714-8845, Fax: 1-607255-9004, Mailing Address: School of Civil and Environmental Engineering; 205 Hollister Hall; Ithaca, NY, USA 14853

Preprint submitted to Journal of Environmental Modelling and Software February 17, 2017

(C) 2017. This manuscript version is made available under the Elsevier user license http://www.elsevier.com/open-access/userlicense/1.0/ 
direct policy search, tipping points, robustness, deep uncertainty

\section{Highlights}

- Managing socio-ecological systems with tipping points is a challenging multi-objective problem

- Direct policy search (DPS) is a computationally efficient method for solving such problems

- Compared to open loop control DPS finds policies that are more robust to deep uncertainties

\section{Software and Data Availability}

- Description and Availability: The Lake Problem optimization code, MORDM re-evaluation code, and best final reference sets are available on Github at https://github.com/julianneq/Lake_Problem_DPS. The optimization and hypervolume calculations can be replicated using the software code available for the Borg MOEA (http://borgmoea.org/), pareto.py (https://github.com/matthewjwoodruff/pareto.py) and the MOEA framework (http://moeaframework.org/).

- Developer: The simulation code was adapted by Julianne Quinn from code developed by Riddhi Singh with adaptations by Tori Ward, Dave Hadka and Jon Herman.

- Funding Source: Development of the code was partially supported by the National Science Foundation, through the Network for Sustainable Climate Risk Management (SCRiM) under NSF cooperative agreement GEO-1240507 as well as the Penn State Center for Climate Risk Management.

- Source Language: The optimization code is written in $\mathrm{C}++$ and the re-evaluation code in Python. 
- License: GNU Lesser General Public License, Version 3

\section{Introduction}

As economic development continues globally, severe ecological consequences of human actions are manifesting themselves in many forms. Altered nutrient cycling, shifting biomes, and decreased biodiversity are just a few examples of the repercussions of anthropogenic activities [46]. More responsible socioecological management will require balancing conflicting objectives, some of which exhibit uncertain and precarious threshold behavior (e.g., Werners et al. 61, Keller et al. 30). For example, we are currently balancing a severe tradeoff between increasing energy production using fossil fuels and avoiding potentially irreversible ecological damages from crossing a threshold atmospheric $\mathrm{CO}_{2}$ concentration [56]. In fact, [41] highlight eight components of the Earth System that could reach catastrophic tipping points as a result of global warming, with the areal extent of Artic summer sea-ice and the Greenland ice sheet facing the most imminent threat.

In environmental systems with thresholds, balancing conflicts in societal values or objectives is further complicated by severe uncertainties associated with identifying thresholds as well as the consequences of crossing them [40, 29]. These uncertainties are often considered "deep" or Knightian uncertainties, meaning planners cannot agree on prior probability density functions to describe the parameters of the system model, or even on the model itself $[36,31]$. In these cases, it is desirable to find robust management plans that perform well across a broad range of possible system conditions [25, 33].

Since its seminal inception $[3,35,59,39]$, the field of decision making under deep uncertainty has emphasized a transition from classical "predict then act" risk management frameworks to exploratory modeling frameworks (e.g., see Dessai et al. 16). These methods move beyond planning for a single expected future and instead emphasize investigating the response of system management

plans to a wide range of deeply uncertain states-of-the-world (SOWs) in order to 
discover robust actions for avoiding unacceptable outcomes $[8,38,23]$. In their recent review, [25] highlight the rapid growth in new methodologies and applications of decision analysis frameworks focused on robustness or deep uncertainty, such as robust decision making (RDM) [38], dynamic adaptive policy pathways [20], many-objective robust decision making (MORDM) [28], and decision scaling [7]. Despite the growing diversity of robustness-focused frameworks, the taxonomy of methods presented by [25] emphasizes the commonalities between them and the importance of bridging their capabilities to advance the field. These approaches share four core methodological components: (1) eliciting or searching for alternative management actions, (2) using exploratory modeling to broadly sample possible SOWs that could impact the performance of alternative policies or actions, (3) eliciting robustness measures that distinguish SOWs of concern, and (4) potentially using sensitivity analysis to clarify the key factors that most strongly influence robustness for subsequent monitoring [25].

This study advances the MORDM framework [28] with a specific focus on two technical contributions: (1) demonstrating the value and use of direct policy search (DPS) [48] for identifying adaptive robust operational control strategies for socio-ecological systems and (2) demonstrating how nonlinear environmental thresholds, or tipping points, pose fundamental challenges for balancing economic benefits and their consequent risks to socio-ecological systems. As initially developed by [28], the MORDM framework focuses on aiding decision makers and stakeholders in learning how to frame complex, ill-defined environmental planning problems and in discovering robust decisions that perform well across a broad array of possible SOWs. A distinguishing feature of MORDM relative to other frameworks is its use of many-objective evolutionary optimization to identify approximately Pareto optimal management decisions. Pareto optimal, or non-dominated, decisions represent those management actions for which improvement in one objective is only possible with degrading performance in one or more other objectives [45]. These solutions are first discovered through multi-objective optimization to one's best estimate of the true SOW. The solutions are then re-evaluated in alternative SOWs to determine how robust they 
are to uncertainties in system parameters. At its core, the MORDM framework provides a posteriori decision support, meaning it first presents explicit representations of key system tradeoffs and robustness challenges and then elicits stakeholder preferences in selecting management actions (i.e., generate first, choose later, as classified by [13]).

In this study, we demonstrate the value of exploiting DPS in the MORDM framework using the classical shallow lake problem [11]. In this didactic example, a hypothetical town situated on a lake attempts to balance the economic benefits it receives from discharging phosphorus $(\mathrm{P})$ into the lake with the environmental costs of irreversibly tipping the lake into a eutrophic state. The behavior of this stylized model of lake eutrophication is representative of many socio-ecological systems with tipping points, such as harvested fish populations, grasslands consumed by cattle on rangelands, and global carbon cycle dynamics $[10,2]$. Early work on the lake problem (e.g., Carpenter et al. 11, Lempert \& Collins 36) has focused on optimizing the town's pollution control policy to maximize the expected net present value of a utility function which rewards economic benefits and penalizes pollution using a monetary valuation of displaced ecological benefits. Collapsing these objectives into a single expected utility function poses several problems. First, it assumes a priori knowledge of stakeholders' values, and agreement among stakeholders on those values. Monetizing environmental benefits and costs to find a single "optimal" solution can fail to capture the full range of achievable objective values, which would better embody the range of preferences among different stakeholders (see, e.g., Admiraal et al. 1). Second, maximizing the expected value of a utility function requires agreement on the probability distribution of stochastic inputs, which poses severe challenges for systems with deeply uncertain characteristics [36, 31].

Recent many-objective extensions of the lake problem have sought to explicitly capture the tradeoffs between economic and environmental objectives (e.g., Singh et al. 54, Hadka et al. 21, Ward et al. 60), as well as deep uncertainty in the lake model parameters (e.g., Singh et al. 54, Hadka et al. 21). [60] find that when optimizing pollution control strategies for the town as a time series 
of $\mathrm{P}$ release decisions, several state-of-the-art multi-objective evolutionary algorithms (MOEAs) fail to find effective policies due to the high dimensional decision space for candidate pollution control action, weak system responses to late period decisions (i.e., temporal salience structure as discussed by [58]), and the non-linear pollution threshold. One way to potentially overcome these challenges is to employ a closed loop control method in which knowledge of the system state is used as a feedback control to inform the decision at each time step [5]. Not only can the additional information provided at each time step improve the signal of the late-period decisions, but it can also allow for a different set of $\mathrm{P}$ release decisions under different realizations of stochastic $\mathrm{P}$ inflows. The open loop intertemporal pollution control strategy employed by [60], however, only finds one vector of pollution control decisions that perform best in expectation, and is reflective of the methodologies used in many environmental policy studies (e.g., Nordhaus 44).

Here, we employ a closed loop control strategy called direct policy search (DPS), that has proven to be a simple and computationally efficient but effective method for solving challenging reservoir control problems [18]. In DPS, the parameters of a function mapping the system state(s) to decisions are optimized rather than the decisions themselves. What results from optimization with DPS is therefore not a sequence of decisions, but a policy that one can operate. DPS is also called parameterization-simulation-optimization in the water resources literature [32], as it involves parameterizing policies, simulating them, and then optimizing their parameters so that they perform best under simulation. In constrast to stochastic dynamic programming (SDP), a commonly used closed loop stochastic control method, DPS can more easily include multiple state variables in the optimized policies, and simulate them over a range of stochastic inputs during the optimization without building an explicit transition probability model. This drastically reduces the curse of dimensionality and allows direct use of system simulations [18], a shortcoming of SDP. Additionally, the DPS simulation model can be coupled with an MOEA to directly optimize multiple objectives. The computational efficiency of DPS makes the method an 
effective technique for optimizing single and multi-objective control problems for a variety of applications $[19,32,48]$.

Here we demonstrate that the built-in control feedback of DPS, whereby the policies use updated knowledge of the system state to inform decisions, also results in control rules that are robust to deep uncertainties in the system characteristics. [11] show that even in lakes with long records of nutrient inputs and concentrations, major uncertainties remain in the values of parameters describing nutrient dynamics in the lake. Furthermore, uncontrolled nutrient inputs from non-point sources are likely to change with deeply uncertain changes in land use. For these reasons, developing adaptive lake management strategies that are robust to deep uncertainty is a vital objective itself. Given the similarity of the lake problem to many socio-ecological systems, DPS holds promise as an effective method for developing robust multi-objective management plans across a variety of problems. This study demonstrates DPS as valuable method for advancing the field of decision making under deep uncertainty, especially when confronting socio-ecological systems with severe tipping points.

\section{Lake Problem Description}

In this study we consider a pollution control problem in which a town must develop a $\mathrm{P}$ emissions strategy that balances its economic benefits and the quality of the lake. We model the $\mathrm{P}$ dynamics in the lake using a theoretical model of lake nutrient dynamics developed by [11] in which water quality can transition between two states: (i) an oligotrophic equilibrium and (ii) a eutrophic equilibrium. Transitions between these states have been observed in many shallow lakes, such as Tomahawk Lagoon in New Zealand and Lakes Takern and Krakesjon in Sweden [53]. In this study, we adapt a dimensionless version of the [11] model used to abstract phosphorus (P) dynamics in Lake Mendota, Wisconsin:

$$
X_{t+1}=X_{t}+a_{t}+Y_{t}+\frac{X_{t}^{q}}{1+X_{t}^{q}}-b X_{t}
$$


where

$X$ is the normalized concentration of $\mathrm{P}$ in the lake,

$a$ are anthropogenic (controlled, point source) P inputs,

$Y \sim L N\left(\mu, \sigma^{2}\right)$ are natural (uncontrolled, non-point source) $\mathrm{P}$ inputs modeled as coming from a log-normal distribution with mean $\mu$ and variance $\sigma^{2}$,

$q$ is a parameter controlling the rate at which $\mathrm{P}$ is recycled from the sediment, $b$ is a parameter controlling the rate at which $\mathrm{P}$ is lost from the lake, and

$t \in\{0,1,2, \ldots\}$ is the time index in years.

The term $\frac{X^{q}}{1+X^{q}}$ represents the inputs of $\mathrm{P}$ recycled from the sediment as a function of the current $\mathrm{P}$ level, $X$. These inputs follow a sigmoid curve whose maximum steepness is controlled by $q$. Larger values of $q$ result in steeper curves and therefore more sudden changes in recycling rates as a function of lake $\mathrm{P}$ concentration. The term $b X$ represents the losses of $\mathrm{P}$ through, for example, outflow or sediment adsorption. Losses are assumed to be a linear function of the lake $\mathrm{P}$ concentration, with larger values of $b$ resulting in greater losses [11].

A graphical depiction of the lake model for different values of $b$ and $q$ is shown in Figure 1, where the temporal fluxes of $\mathrm{P}$ into and out of the lake are plotted as a function of the current concentration of $\mathrm{P}$ in the lake, $X_{t}$. The black line in Figure 1(a) shows the rate of losses for $b=0.4$, while the colored curves show the rate of $\mathrm{P}$ inputs from recycling under different values of $q$. In Figure $1(\mathrm{~b})$, the black curve shows inputs from recycled $\mathrm{P}$ when $q=2.5$, while the colored lines show the losses of $\mathrm{P}$ for different values of $b$. Inputs from anthropogenic and natural $\mathrm{P}$ are assumed to be 0 in this figure; adding them would vertically translate the recycling curves. 

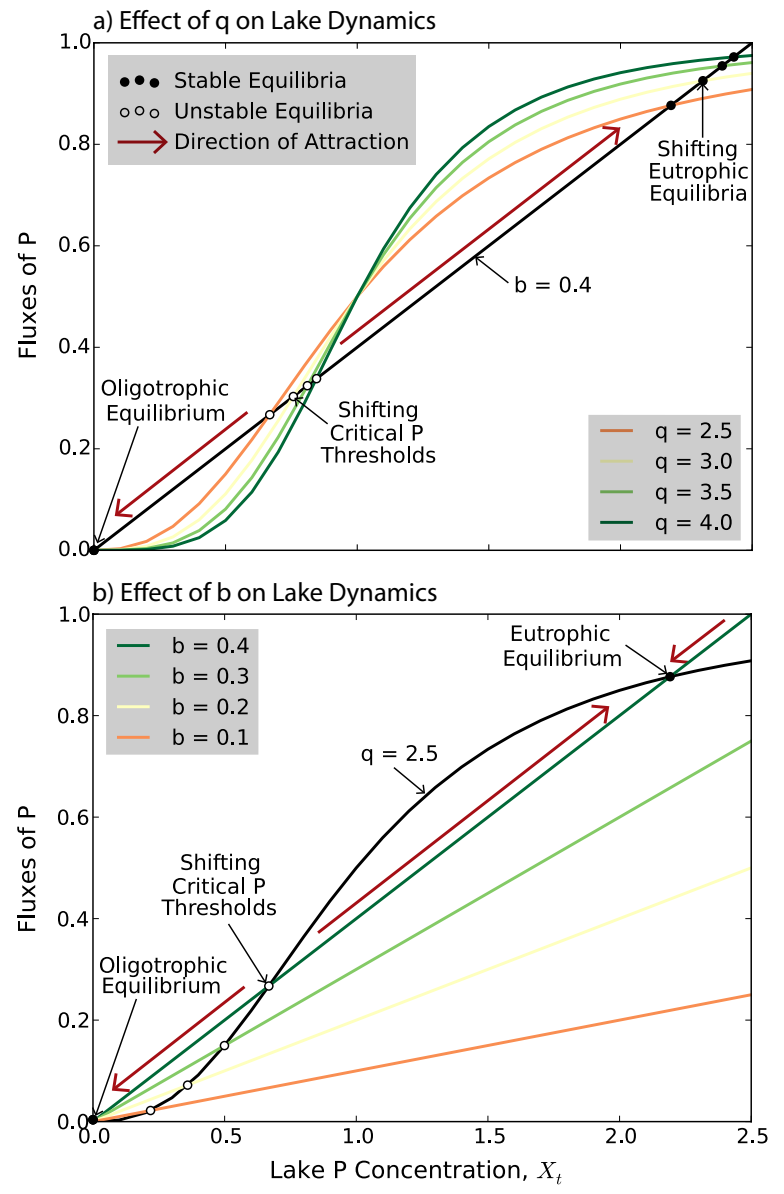

Figure 1: Non-linear dynamics of the irreversible lake model. Curves indicate inputs of $\mathrm{P}$ recycled from the sediment and lines indicate $\mathrm{P}$ sinks. The system is in equilibrium when the $\mathrm{P}$ inputs and sinks are equal, which occurs at three locations for each combination of inputs and sinks functions. Two of these intersections are stable equilibria, and the third in the middle is unstable. The unstable equilibrium is referred to as the "critical P threshold," as crossing it moves the system to an irreversibly eutrophic state for the parameters of $b$ and $q$ investigated in this study. Panel (a) shows the effect of $q$ on the location of the critical P threshold, while panel (b) shows the effect of $b$. Higher values of both $b$ and $q$ result in higher critical $\mathrm{P}$ thresholds, making it easier to avoid moving the system from a stable oligotrophic equilibrium to a stable eutrophic equilibrium. 
Note that for the alternate values of $b$ and $q$ plotted in Figures 1(a) and (b), each of the curves representing inputs of recycled $\mathrm{P}$ and the lines representing outputs from $\mathrm{P}$ sinks intersect at three different $\mathrm{P}$ concentrations. At each of the points of intersection, the inputs are equal to the outputs and the net flux of $\mathrm{P}$ is 0 , meaning the lake's $\mathrm{P}$ concentration is in equilibrium. When the sources are greater than the sinks, the $\mathrm{P}$ concentration tends to increase, and when the sinks are greater, it tends to decrease. These dynamics result in two stable equilibria shown in black that are separated by an unstable equilibrium shown in white. The stable equilibrium that occurs at a low $\mathrm{P}$ concentration represents a healthy, oligotrophic equilibrium, while that at a high $\mathrm{P}$ concentration represents an unhealthy, eutrophic equilibrium.

If the concentration of $\mathrm{P}$ in the lake exceeds the unstable equilibrium in any of the cases plotted above, it will be impossible for the lake to return to an oligotrophic equilibrium unless $\mathrm{P}$ is actively removed from the system. Reducing $\mathrm{P}$ inputs alone will not be sufficient, as this figure already shows the system with 0 anthropogenic and natural $\mathrm{P}$ inputs. These sample lake model parameterizations consequently possess "irreversible" $\mathrm{P}$ thresholds, or tipping points, that pose severe management challenges. Such lakes tend to be shallow, located in P-rich regions, or receive sustained high $\mathrm{P}$ inputs [11]. Because crossing the unstable equilibrium results in a permanently polluted lake, we call the $\mathrm{P}$ concentration at this point the "critical $\mathrm{P}$ threshold."

As Figure 1 shows, different lake parameterizations can have profound implications for how one manages the system, as the value of the critical P threshold can vary greatly for different values of $b$ and $q$. For a given loss rate, $b$, higher values of $q$ result in higher critical $\mathrm{P}$ thresholds, allowing managers to emit more $\mathrm{P}$ before the lake enters a permanently polluted state. However, the slope of the recycling curve is steeper for larger values of $q$, making the transition across this threshold more abrupt. For a given value of $q$, the same is true; larger values of $b$ result in higher critical $\mathrm{P}$ thresholds but often with a faster transition across them.

Furthermore, the parameters describing the lake's dynamics can be difficult 
to estimate from empirical data. [11] try to do so with 21 years of data on $\mathrm{P}$ input rate and mass in Lake Mendota. After generating point estimates of the model parameters, they generate bootstrapped parameter estimates by adding back randomly selected residuals and find that reasonable estimates for the characteristics of the lake dynamics span the three main possibilities of reversible, hysteretic, and irreversible. This uncertainty severely challenges the development of effective management strategies. Even in modeled settings, reliable estimates of model parameters often cannot be made until a threshold has already been crossed (see, e.g., Lempert \& Collins 36), at which point it is too late to inform management if the system is irreversible.

Here, we investigate how to formulate effective management policies for a system with assumed values of model parameters related to lake dynamics and economic discounting, but then test how robust these policies are to parametric uncertainty in the case that the assumed values are incorrect, applying a robustness method called Multi-Objective Robust Decision Making (MORDM; Kasprzyk 27). While more robust solutions may potentially be discovered by initially optimizing to the deeply uncertain SOWs, this improved robustness may result in large regrets if the system never confronts the most extreme SOWs. In MORDM, the sampled ranges of uncertain parameters are subjectively chosen to be wide enough to discover failures and it could be that some of the sampled SOWs are extremely unlikely. Following Kasprzyk [27], we implement MORDM to establish an initial baseline of performance of alternative management plans. In discovering robust baseline solutions, we implicitly minimize regret to an assumed SOW representing the best current state of knowledge. Once key uncertainties driving system performance are discovered, more robust policies can be developed by monitoring these uncertainties and adapting the policies as more information becomes available. 


\section{Optimization Problem}

\subsection{Lake Management Objectives}

The general challenge we consider in this study is how much anthropogenic $\mathrm{P}, a_{t}$, to emit over time in order to simultaneously optimize multiple, conflicting objectives with respect to environmental and economic goals. Because the objectives are conflicting, tradeoffs exist between different lake management plans. We find the set of "non-dominated" management plans, meaning that among the solutions in the set, no solution does better than another in all objectives. This set is called the Pareto set.

Following [60], we consider four objectives listed in Table 1 and described below.

Table 1: Performance Objectives of Lake Pollution Control Problem

\begin{tabular}{llcc}
\hline Objective & Description & Min/Max & Epsilon \\
\hline Economic Benefits & $\begin{array}{l}\text { Discounted economic benefits, assumed pro- } \\
\text { portional to discounted P emissions }\end{array}$ & Max & 0.01 \\
Lake P Concentration & $\begin{array}{l}\text { Measure of the lake quality, where lower P } \\
\text { concentrations correspond to clearer lakes }\end{array}$ & Min & 0.01 \\
Policy Inertia & $\begin{array}{l}\text { Measure of the stability of the control policy, } \\
\text { where stable policies are favored }\end{array}$ & & 0.0001 \\
Reliability & $\begin{array}{l}\text { Percentage of time that the lake P concentra- } \\
\text { tion is below the critical P threshold, } X^{\text {crit }}\end{array}$ & & \multirow{2}{*}{ Max } \\
\end{tabular}

1. Maximize expected economic benefits: In each of $N$ simulations of $T$ years of random natural $\mathrm{P}$ inflows, the discounted economic benefits are calculated as $\sum_{t=0}^{T-1} \alpha a_{t, i} \delta^{t}$, where $\alpha$ is a dimensionless parameter representing the town's willingness to pay for pollution, $\delta$ is the discount factor used to convert future benefits to present benefits, and $a_{t, i}$ is the anthropogenic $\mathrm{P}$ release in the $t^{t h}$ year of the $i^{t h}$ simulation. The expected economic benefits, $O_{1}$, are the average economic benefits across the $N$ 
simulations of $T$ years:

$$
O_{1}=\frac{1}{N} \sum_{i=1}^{N}\left(\sum_{t=0}^{T-1} \alpha a_{t, i} \delta^{t}\right)
$$

As in [60], we use $\alpha=0.4$ and $\delta=0.98$.

2. Minimize worst case average $\mathbf{P}$ concentration: $\mathrm{In}$ each of $T$ years, the average $\mathrm{P}$ concentration across $N$ simulations is calculated. For water quality purposes, the lake manager seeks to minimize the maximium of these $\mathrm{T}$ averages, $\mathrm{O}_{2}$ :

$$
O_{2}=\max _{t \in(1, \ldots, T)} \frac{1}{N} \sum_{i=1}^{N} X_{t, i}
$$

where $X_{t, i}$ is the lake $\mathrm{P}$ concentration in the $t^{t h}$ year of the $i^{t h}$ simulation.

\section{Maximize average inertia of $\mathbf{P}$ control policy: Since rapid $P$} reductions require large investments in infrastructure such as tertiary treatment, the lake manager would like to best maintain policy inertia, or stability. Here inertia, $O_{3}$, is defined as the average fraction of $T-1$ time steps across $N$ simulations that require reductions of less than some limit, $I_{\text {limit }}$ :

$$
O_{3}=\frac{1}{N} \sum_{i=1}^{N}\left(\frac{1}{T-1} \sum_{t=1}^{T} \phi_{t, i}\right) \text { where } \phi_{t, i}= \begin{cases}1, & a_{t-1, i}-a_{t, i}<I_{\text {limit }} \\ 0, & a_{t-1, i}-a_{t, i} \geq I_{\text {limit }}\end{cases}
$$

As in [60], we use a value of 0.02 for $I_{\text {limit }}$. In a real system, the value of $I_{\text {limit }}$ should be elicited from stakeholders, who would have to evaluate the costs and benefits associated with these reductions. 


\section{Reliability of policy in staying below the lake's critical $\mathbf{P}$}

threshold: Because the lake is irreversible and crossing its critical $\mathrm{P}$ threshold, $X^{\text {crit }}$, results in permanent eutrophication, the lake manager seeks to maximize the average fraction of time the lake is below this threshold. This is defined as the policy's reliability, $O_{4}$ :

$$
O_{4}=\frac{1}{N T} \sum_{i=1}^{N}\left(\sum_{t=1}^{T} \theta_{t, i}\right) \text { where } \theta_{t, i}= \begin{cases}1, & X_{t, i}<X^{\text {crit }} \\ 0, & X_{t, i} \geq X^{c r i t}\end{cases}
$$

The reliability is constrained to be at least $85 \%$. It was challenging for several MOEAs to find solutions meeting this constraint in the prior work by $[60]$.

In this study, we compare two solution strategies for optimizing multiobjective lake management control policies: 1) open-loop control via intertemporal optimization and 2) closed loop control via direct policy search.

\subsection{Solution Strategies}

\subsubsection{Intertemporal Open Loop Control}

Following prior work on multi-objective optimization of the lake problem $[54,21,60]$ and common practice in the integrated assessment literature (e.g., Nordhaus 44), we use an open loop intertemporal solution strategy in which there are $T$ decision variables, $a_{t}$, representing the anthropogenic discharges at each time step $t \in(0,1, \ldots, T-1)$. Drawing on the objectives defined in equations (2)-(5), the intertemporal optimization problem is thus defined as:

$$
\begin{gathered}
\text { Minimize } F(a)=\left(-O_{1}, O_{2},-O_{3},-O_{4}\right) \\
\quad a=\left(a_{0}, a_{1}, \ldots, a_{T-1}\right)
\end{gathered}
$$


Subject to:

$$
\begin{gathered}
O_{4} \geq 0.85 \\
0.01 \leq a_{t} \leq 0.1 \forall t
\end{gathered}
$$

Under this solution strategy, $T$ anthropogenic $\mathrm{P}$ emissions, $a_{t}$, are optimized to maximize expected economic benefits, minimize maximum average lake $\mathrm{P}$ concentration, maximize inertia, and maximize reliability. The same $T$ releases are made in each of the $N$ simulations, such that $a_{t, i}=a_{t} \forall i$. The only constraints are that the reliability be at least $85 \%$ (Equation 8 ), and that $\mathrm{P}$ emissions range between 0.01 and 0.1 so that economic activity is relatively stable (Equation 9 ).

This is an open loop control strategy because the output (the resulting lake $\mathrm{P}$ concentration) does not feed back into the control (the $\mathrm{P}$ release decision). All decisions are determined based on the initial state and the model, with the goal of finding a solution that performs best on average across a range of realizations of random natural $\mathrm{P}$ inflows. This is a characteristic of most intertemporal optimization strategies if used without re-optimization. The second control strategy, direct policy search, employs closed loop control in which information on the system state, in this case the $\mathrm{P}$ level in the lake, feeds back to the control, thus enabling different $\mathrm{P}$ release decisions in different realizations [5]. For a depiction of the differences between open and closed loop optimization, see Figure A1 in the appendix.

\subsubsection{Direct Policy Search Closed Loop Control}

The second solution strategy, termed direct policy search (DPS) [48], is a closed loop control method that does not optimize the anthropogenic P releases, $a_{t}$, themselves, but rather the parameters of a state-aware control rule. Also known as parameterization-simulation-optimization [32], DPS has been used for several single objective applications in the water and environmental literature [19, 32]. More recently, DPS applications have expanded their focus toward better addressing uncertainties and multiple objectives, mainly in reservoir operations $[14,18,49]$. The present study builds on this prior work by 
introducing DPS into the socio-ecological tipping points literature $[54,10,60]$ and demonstrating the value of its state-based feedback control for navigating deeply uncertain threshold management tradeoffs [54, 21].

As applied to the lake problem, candidate DPS control rules are represented as functions mapping the current state of the lake's $\mathrm{P}$ concentration, $X_{t}$, to a $\mathrm{P}$ release decision, $a_{t}$. Figure 2 illustrates an example of such a control policy function. At low $\mathrm{P}$ concentrations, one would expect to be able to release high amounts of $\mathrm{P}$ without worrying about crossing the critical $\mathrm{P}$ threshold. However, as one approaches this threshold (shown in red), the P release should decrease to ensure high reliability. If the $\mathrm{P}$ concentration is above the critical $\mathrm{P}$ threshold, however, reliability can no longer be improved since the lake is irreversible, so it may be best to increase $\mathrm{P}$ releases again to maximize further economic benefits.

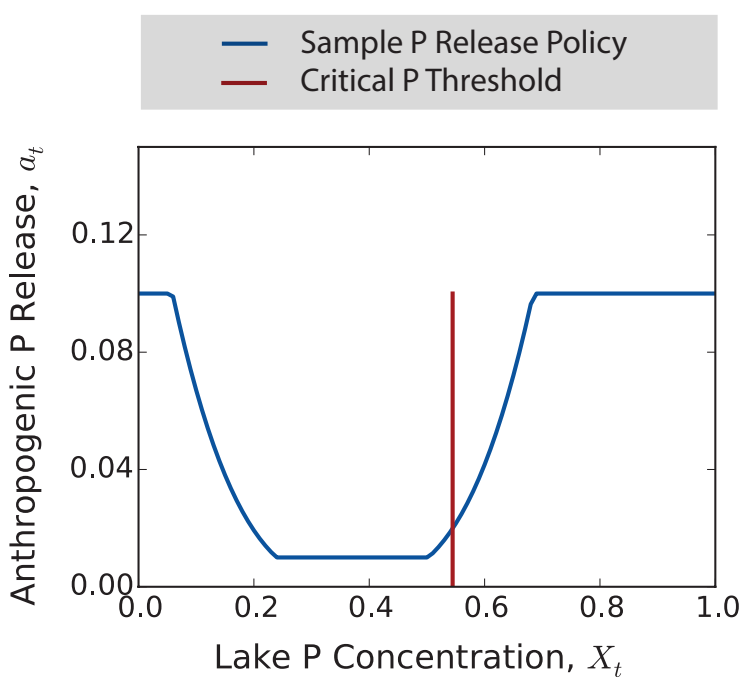

Figure 2: Sample P release policy. The blue curve indicates the amount of anthropogenic $\mathrm{P}, a_{t}$, released as a function of the current lake $\mathrm{P}$ concentration, $X_{t}$. The red line is the critical $\mathrm{P}$ threshold, $X^{\text {crit }}$, above which the system is irreversibly eutrophic.

In this study, we use cubic radial basis functions to define the function 
mapping lake $\mathrm{P}$ concentrations to $\mathrm{P}$ release decisions, as many studies have shown them to be effective universal approximators for DPS, generalizing well when operating a system on out-of-sample inputs (e.g., Buşoniu et al. 9, Giuliani et al. 18). This optimization problem is formulated as:

$$
\begin{gathered}
\text { Minimize } F(a)=\left(-O_{1}, O_{2},-O_{3},-O_{4}\right) \\
a=\left(a_{0, i}, a_{1, i}, \ldots, a_{T-1, i}\right) \\
a_{t, i}=\min \left(\max \left(\sum_{j=1}^{n} w_{j}\left|\frac{X_{t, i}-c_{j}}{r_{j}}\right|^{3}, 0.01\right), 0.1\right) \forall t, i
\end{gathered}
$$

Subject to:

$$
\begin{aligned}
& O_{4} \geq 0.85 \\
& -2 \leq c_{j} \leq 2 \\
& 0 \leq r_{j} \leq 2 \\
& 0 \leq w_{j} \leq 1 \\
& \sum_{j=1}^{n} w_{j}=1
\end{aligned}
$$

where $c_{j}, r_{j}$ and $w_{j}$ are the centers, radii and weights of $n$ cubic radial basis functions. The decision variables are these $3 n$ parameters, rather than the $T$ decision variables $a_{t}$ in the intertemporal optimization. For this problem, we use $n=2$, resulting in 6 decision variables compared to $T=100$ decision variables using the intertemporal solution strategy. Notice that with this solution strategy, different $\mathrm{P}$ release decisions, $a_{t, i}$, can be made in each of the $N$ simulations, as the decisions are informed by the different resulting lake $\mathrm{P}$ concentrations, $X_{t, i}$.

DPS has proven to be a powerful and computationally efficient method for solving many-objective stochastic control problems [18]. Unlike the open loop intertemporal strategy, DPS is able to incorporate information about the lake's state in each stochastic scenario to guide more adaptive and responsive $\mathrm{P}$ release decisions. Additionally, one could easily incorporate more information into the 
DPS policies. For example, the above formulation could include the lake $\mathrm{P}$ concentrations in both the current and previous time steps. For simplicity, we demonstrate the method with just a single input. Note that this simple formulation assumes that one can perfectly measure the lake $\mathrm{P}$ concentration before each release decision and that the presence of an irreversible tipping point is known. In future extensions, we will explore more advanced data assimilation strategies that can account for observational error and more complex learning (e.g., about the model parameters).

\subsection{Optimization Algorithm}

We use the intertemporal and DPS solution strategies to solve the multiobjective formulation of the lake problem for $T=100$ years and $N=100$ realizations of randomly generated natural $\mathrm{P}$ inflows, initializing the lake concentration at $X_{0}=0$. Values of the lake model parameters are given in Table 1. The slope of the losses curve, $b$, is set to 0.42 , and the shape parameter of the recycling curve, $q$ is set to 2.0. These parameters correspond to a lake with a critical $\mathrm{P}$ threshold, $X^{\text {crit }}$, of 0.54 . Natural $\mathrm{P}$ inflows are simulated from the same distribution as in Scenario 2 from [60]: a log-normal distribution with a real-space mean of $\mu=0.03$ and variance of $\sigma^{2}=10^{-5}$. [60] show that this scenario poses a severe challenge to six state-of-the-art MOEAs in finding Pareto-approximate lake management strategies.

MOEAs are heuristic optimization algorithms that use an iterative search process to modify and evolve a population of candidate solutions using a number of different probabilistic operators for mating, mutation, selection, and archiving [47]. They are particularly effective at solving nonlinear, nonconvex, multimodal and discrete multi-objective problems where derivative-based algorithms often fail [43]. While MOEAs have emerged as dominant solution tools in the environmental literature [43, 47], of the six state-of-the-art MOEAs tested by [60] on the intertemporal lake problem formulation, only the Borg MOEA [22] was able to consistently find high quality tradeoff solutions. [60] attributed Borg's success to its use of $\varepsilon$-dominance archiving, $\varepsilon$-progress and multiple self- 
adaptive search operators.

The $\varepsilon$-dominance, or block dominance, concept speeds up algorithmic search processes relative to traditional point dominance while also providing a mathematical proof of convergence and guaranteeing a diverse representation of problems' tradeoffs [34]. For each objective, a preferred precision level, $\varepsilon$, is specified by the user, creating multi-dimensional $\varepsilon$-boxes [22]. Within each $\varepsilon$-box, only the solution closest to the ideal point of that box is stored in the Pareto-approximate set, or "archive," throughout the search, even if multiple non-dominated solutions have been discovered within that box. This thins the number of solutions stored in the archive, preventing a computationally burdensome growth in the number of solutions that must be sorted each iteration $[22,34]$. In the Borg MOEA, $\varepsilon$-progress measures how frequently a solution is added to the archive that improves upon a previous solution by $>\varepsilon$, meaning the new solution lies within an $\varepsilon$-box that dominates the $\varepsilon$-box in which the previous solution resided [22]. In the Borg MOEA, the probability of using algorithm operators that frequently contribute to $\varepsilon$-progress increases adaptively throughout the search, while the probability of using operators that do not frequently contribute to $\varepsilon$-progress decreases. This makes the algorithm largely insensitive to the initial values of its parameters, unlike the other algorithms tested by [60]. For these reasons, we also use the Borg MOEA as our solver of choice.

\subsection{Evaluation of Solution Strategies}

We use the hypervolume metric to compare the intertemporal and DPS solution strategies in terms of algorithmic efficiency and effectiveness in attaining high quality approximations of the problem's Pareto frontier. Hypervolume is a measure of the multi-dimensional "volume" of space dominated by a set of Pareto-approximate solutions [62]. One can calculate the hypervolume of the best-known approximation of the Pareto set, or "reference set," after different numbers of function evaluations (NFE) throughout the search process and observe how quickly the different solution strategies are able to find good solutions. Additionally, the hypervolume metric is useful for comparing the final 
performance of different solution strategies. Here, we use it to distinguish and compare the relative quality of the best-known Pareto sets of the intertemporal and DPS solution strategies.

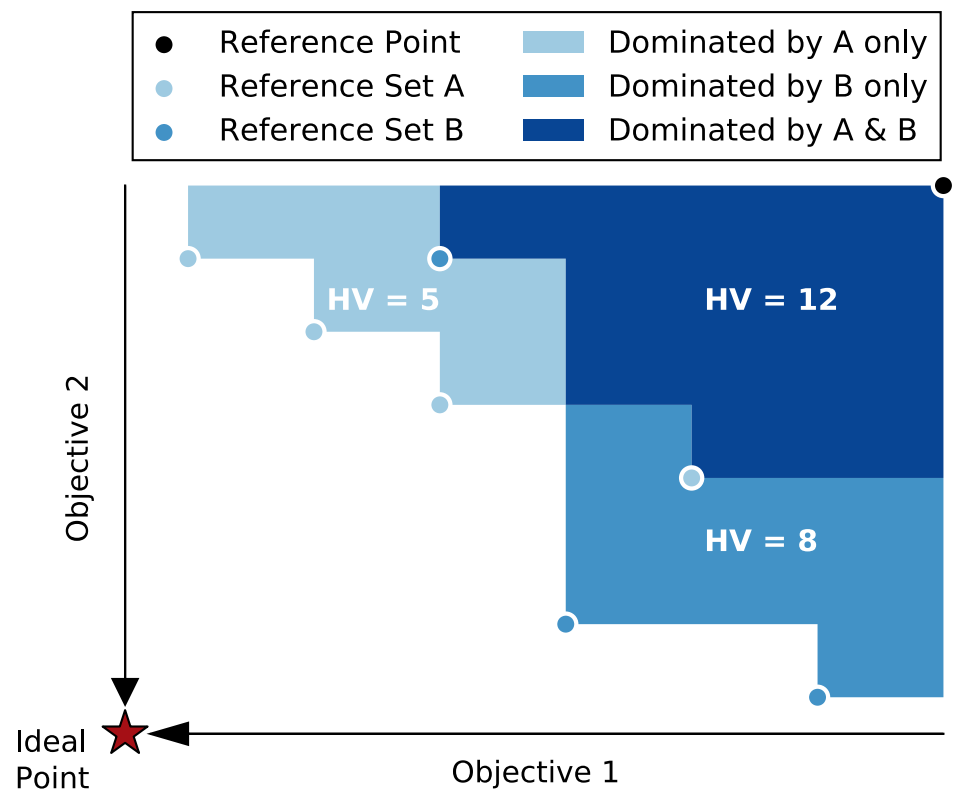

Figure 3: Sample hypervolume comparison. Light blue points represent solutions found by method A, while dark blue points represent solutions found by method B. The solutions found by method B dominate a greater area, or hypervolume, indicating that this method performs better than method A.

Figure 3 illustrates how hypervolume (HV) can be used for such a comparison. This figure shows sample reference sets from two solution strategies, A and $\mathrm{B}$, on a two-objective minimization problem. The $\mathrm{HV}$ of each reference set is therefore an area calculated with respect to a reference point. One can see that two solutions in reference set $\mathrm{B}$ dominate one solution in reference set $\mathrm{A}$, while the third solution in reference set $\mathrm{B}$ is dominated by three points in reference set A. Looking at the hypervolume metric, though, one can see that the reference set from method B dominates a larger space $(\mathrm{HV}=8+12=20)$ than the reference 
set from method A $(\mathrm{HV}=5+12=17)$, indicating that method $\mathrm{B}$ is able to find a better approximation to the true Pareto front and is therefore the preferred method.

\section{Computational Experiment}

\subsection{Random Seed Analysis}

Since the Borg MOEA is a stochastic optimization algorithm whose search depends on the random seed used to initialize the population and generate new solutions, we solve the lake problem using 50 different random seed runs with Borg's default initial parameter values [22]. Performing a random seed analysis allows one to control for variability and evaluate the consistency in search performance. In this study, we set the NFE for each seed to 200,000 to provide sufficient time for the Borg MOEA to attain high quality approximations of the tradeoffs using both the intertemporal and DPS solution strategies (see Figure 5 in Section 5.1).

To evaluate the search efficiency of each solution strategy, we calculate the hypervolume of the archive every 1,000 function evaluations for each seed. We then normalize these values by dividing by the best final hypervolume achieved across all seeds and solution strategies. To compare the final performance of each strategy, we find a reference set from each method by aggregating the terminal set of solutions found by each seed from that method and re-sorting them into one Pareto-approximate set per method. We plot these two reference sets on the same axes to visualize the amount of space dominated by the solutions found from each method (see Figure 4 in Section 5.1).

The results of the computational experiment are given in Section 5.1, and the quality of the final solutions analyzed in Section 5.2.

\subsection{Robustness Analysis}

As discussed in our introduction of the lake problem's dynamics in Section 2 , it is an important and realistic concern that assumptions related to the lake 
model's parameters could fundamentally change the performance of alternative pollution control strategies. Many systems like managed lakes are subject to deep parametric uncertainty, meaning decision makers do not know, or cannot agree on, prior probability distributions to describe the potential values of the systems' parameters $[31,35,33]$. In such cases, it is desirable to find "robust" policies that perform well under a broad range of potential system characteristics. Here, we seek to compare the performance of the lake management plans derived by each method under the assumed lake characteristics to which they were optimized, as well as under other deeply uncertain states of the world (SOWs) to which they may actually be applied.

Building on initial work by [28] and several recent extensions [24, 54, 21], we use the many-objective robust decision making (MORDM) framework to evaluate the robustness of the control policies derived from the intertemporal and DPS solution strategies. As mentioned in the introduction, MORDM, like several other methods used to assess robustness, follows four key steps outlined by [25]. The first step is to generate alternative management plans for the robustness analysis. Some methods pre-specify such plans, while MORDM searches for them using multi-objective optimization. In this study, we generate alternative lake management plans using multi-objective optimization with the intertemporal and DPS solution strategies.

The next step in any robustness analysis is to re-evaluate the management alternatives across a broad range of SOWs generated by globally sampling the deeply uncertain factors. The third step is to quantify how well the different management plans generalize across these uncertain SOWs. There are many different measures of robustness used in the literature. [25] highlight four: two satisficing metrics $[57,26]$ and two regret metrics $[36,51]$. In comparing these four metrics on a water portfolio planning problem, [25] find that the domain criterion satisficing measure [57], which quantifies the fraction of SOWs in which desired performance levels are met, is the only metric that prioritizes solutions meeting the stakeholders' minimum performance criteria. For this reason, we also use the domain criterion satisficing metric to quantify robustness in this 
study. However, in a real system we recommend calculating a variety of robustness metrics considering a range of thresholds of acceptability, as stakeholders may favor different metrics or thresholds based on their risk attitudes. Furthermore, the choice of metric or threshold level may influence which solutions are considered most robust, introducing more conflicts that stakeholders should consider in evaluating alternative management plans [25]. On the contrary, different metrics or thresholds may ease tension if similar conclusions are drawn despite being derived under different assumptions of what it means to be robust [23]. In both cases, the additional robustness analysis can aid stakeholders in coming to consensus on candidate actions even if their conclusions emerge for different reasons.

Regardless of which metrics are ultimately used to quantify robustness, the final step employed by several robustness analysis methods including MORDM is a sensitivity analysis to determine which parameters are most important for ensuring acceptable system performance. This is also referred to as scenario discovery, and can be achieved through factor mapping [8, 17], or factor prioritization $[55,50]$. Factor mapping techniques identify parameter ranges or combinations of parameter ranges that lead to poor performance, while factor prioritization ranks the uncertain parameters by importance to system performance. Ideally, managers could use the results of such sensitivity analyses to focus their attention on controlling or monitoring the key parameters driving system performance.

\subsubsection{Evaluation of Alternatives}

For this study, we generate 1,000 alternative SOWs from a Latin hypercube sample across the ranges of all uncertain parameters. All parameter ranges are sampled uniformly. We assume the same deeply uncertain parameters and ranges used by [21] for a robustness analysis of the same four-objective version of the lake problem, considering only irreversible lakes. We also add one uncertain parameter: the initial $\mathrm{P}$ concentration in the lake, $X_{0}$. Table 1 lists the uncertain parameters, their base values in the SOW to which the policies are optimized 
and the ranges over which they are sampled for the robustness analysis. The results of the re-evaluation are given in Section 5.3.1.

Table 2: Ranges of sampled uncertain parameters and their base values. Lake management plans are optimized to a lake modeled by the base values and reevaluated on 1,000 alternative SOWs generated from a Latin hypercube sample across the parameter ranges given in the table. All parameter ranges are sampled uniformly.

\begin{tabular}{clccc}
\hline Parameter & Description & Base Value & Minimum & Maximum \\
\hline$b$ & Linear P loss parameter & 0.42 & 0.1 & 0.45 \\
$q$ & Shape parameter of sigmoid & 2.0 & 2.0 & 4.5 \\
& curve modeling P recycling & & & \\
$\mu$ & Mean natural P inflow & 0.03 & 0.01 & 0.05 \\
$\sigma^{2}$ & Real-space variance of natural P & $10^{-5}$ & $(0.001)^{2}$ & $(0.005)^{2}$ \\
& inflow & & & \\
$X_{0}$ & Initial lake P concentration & 0.0 & 0.0 & 0.3 \\
$\delta$ & Discount parameter for calculat- & 0.98 & 0.93 & 0.99 \\
& ing economic benefits & & & \\
\hline
\end{tabular}

\subsubsection{Calculation of Robustness}

As stated earlier, we quantify the robustness of each lake management plan in terms of the fraction of sampled SOWs in which certain performance criteria are met. For real-world decision-support applications, these criteria are typically elicited from stakeholders (see e.g., Lempert \& Groves 37, Moody \& Brown 42, Herman et al. 24). For illustrative purposes on this stylized model, we simply specify three possible sets of criteria a stakeholder might demand:

1. Average Economic Benefits $>0.2$

2. Average Reliability $>95 \%$

3. Average Economic Benefits $>0.2$ and Average Reliability $>95 \%$ 
For each solution found by DPS and the intertemporal optimization, we calculate the percent of sampled SOWs in which each of the above criteria are met. The results of these robustness calculations are given in Section 5.3.1.

\subsubsection{Sensitivity Analysis}

The final step in MORDM is to use scenario discovery [8] to determine what ranges of model parameters lead to poor performance. Two commonly used factor mapping approaches include the Patient Rule Induction Method, or PRIM [17], and Classification and Regression Trees, or CART [6]. Both of these methods assume poor performance can be explained by independent combinations of input parameter ranges, and therefore fail to capture non-linear interactions between model parameters that can lead to unacceptable performance. Recent approaches have improved upon PRIM by first performing Principal Components Analysis (PCA) and then applying PRIM to the rotated datasets [15]. However, this modification only addresses the orthogonality constraint of PRIM, not its linearity constraint. For this reason, we perform a visual factor mapping sensitivity analysis to identify which joint ranges of lake model parameters cause particular management plans to fail. This approach enables a better treatment of the nonlinear nature of the lake model's irreversible tipping point. The results of this analysis are given in Section 5.3.2.

\section{Results and Discussion}

\subsection{Tradeoffs and Problem Difficulty}

The results of our computational experiment indicate that DPS is able to find more dominant lake management policies than the intertemporal method. Figure 4 shows the final reference sets found using both solution strategies. Here, we obtain the solutions in each method's reference set by combining the results from the 50 random seed runs of the Borg MOEA (see Section 4.1). DPS found

260 non-dominated solutions and the intertemporal optimization found 86 . In Figure 4 , the arrows designate the directions of increasing preference for the 
three primary axes. Larger points designate higher levels of reliability. The star designates the ideal solution, thus larger points closer to the star are favorable. The DPS solutions in blue dominate many of the intertemporal solutions in red, as they perform better in every objective. That is, the DPS solutions are able to achieve greater economic benefits at much lower maximum average $\mathrm{P}$ concentrations while maintaining high levels of reliability and inertia. Figure 4 clearly highlights that the final hypervolume of the management plans found using DPS exceeds that of the management plans found using the intertemporal approach. 
a) Final reference sets
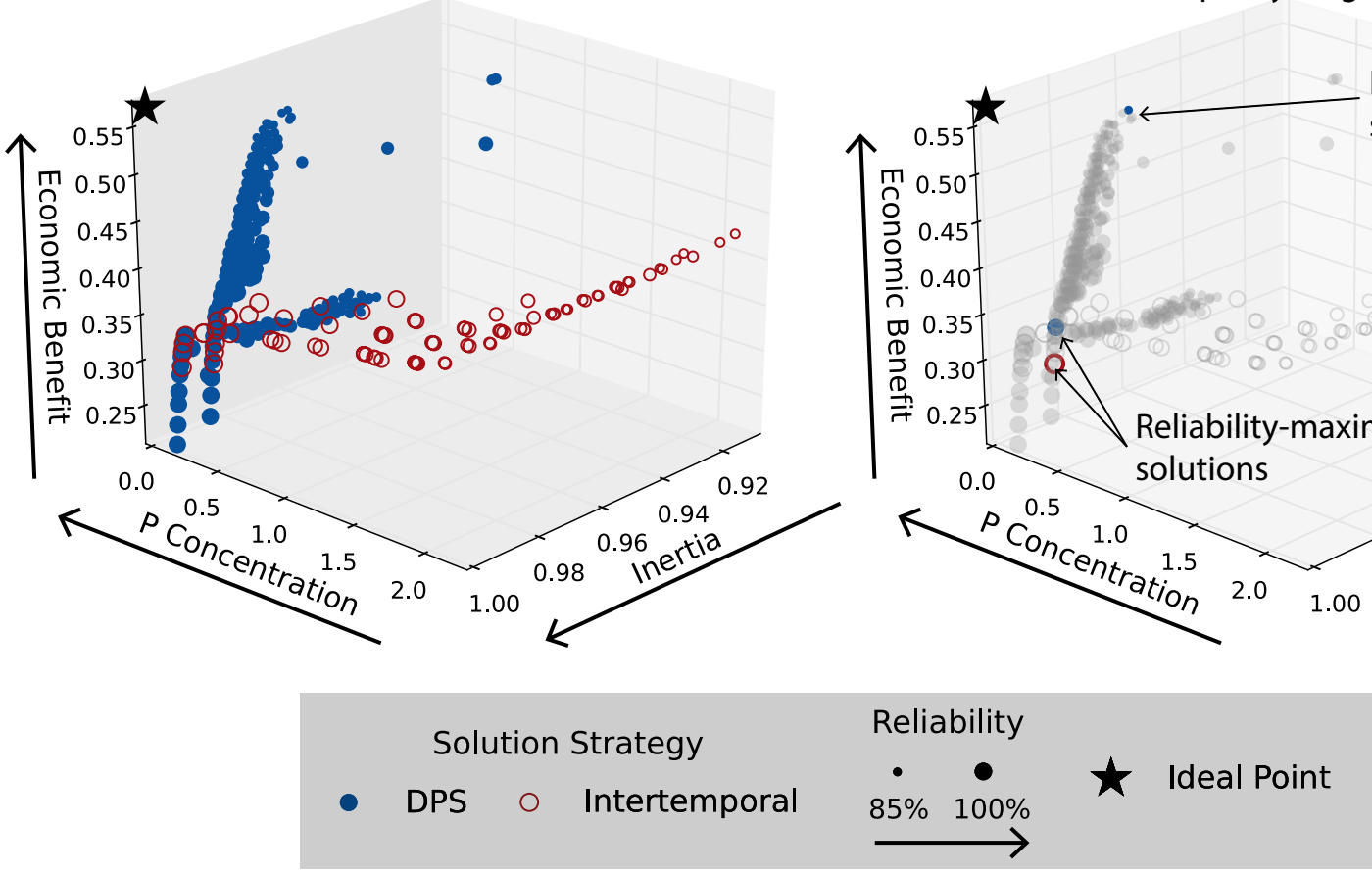

b) Selected solutions for policy diagnostics

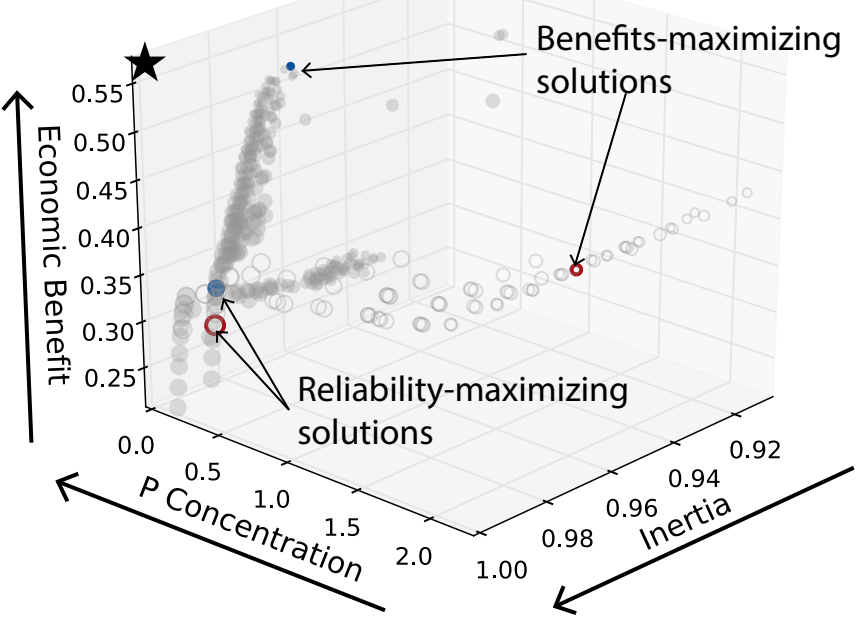

Benefits-maximizing solutions

Figure 4: Pareto-approximate reference sets obtained using the intertemporal (red) and DPS (blue) solution strategies. Arrows indicate direction of preference along each axis. The size of the points represents their reliability, with larger circles preferred. Panel (a) shows that most of the DPS solutions dominate the intertemporal solutions, performing better in every objective. Panel (b) highlights the solutions from each method with the greatest reliability and economic benefits. The decisions resulting from these solutions are examined in further analyses.

In addition to finding better final solutions, the DPS method is able to discover these solutions much faster. Figure 5 shows the relative computational efficiency of each method by plotting the relative hypervolume achieved by each seed versus the number of function evaluations in the search. Relative hypervolume is calculated as a fraction of the space dominated by the best reference 
set achieved by a single seed. The figure shows that all of the DPS seeds in blue converge to near-perfect relative hypervolume. Additionally, these solutions are found very quickly, some within only a few thousand function evaluations and all before 100,000. Using the intertemporal solution strategy, however, the Borg MOEA is unable to generate any hypervolume until about 20,000 evaluations, and while most of the seeds also converge by 100,000 evaluations, they do so to a much lower relative hypervolume (roughly half) with a greater spread across seeds. While it may not be surprising that the DPS solution strategy converges faster than the intertemporal solution strategy since it has far less decision variables, it is noteworthy how much faster it converges, and to what degree it improves upon the attainable system performance. DPS converges so fast that even the initial population of 100 solutions generated from a uniform random sample by the Borg MOEA often includes Pareto-approximate solutions. It was not obvious a priori that such a simple policy rule with only six decision variables would be able to adequately model such effective policies. 


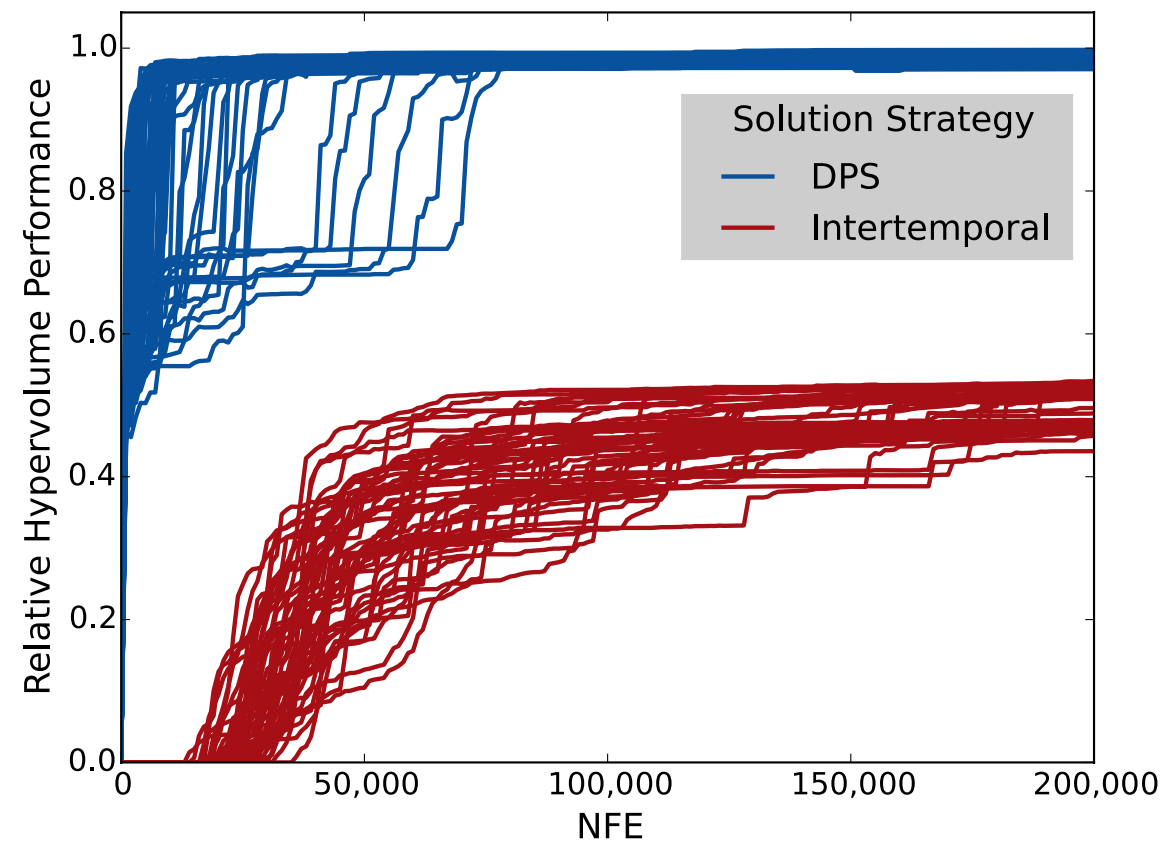

Figure 5: Search convergence using intertemporal (red) vs. DPS (blue) solution strategies. Each line depicts the relative hypervolume vs. the number of function evaluations (NFE) for a different random seed of the Borg MOEA, where hypervolume is given as a fraction of the best-known Paretoapproximation set. Greater hypervolume values are preferred. Across all seeds, the DPS solution strategy is able to generate much greater hypervolume significantly faster.

The difficulty of solving the intertemporal version of the lake problem illustrated in Figure 5 is consistent with the prior published MOEA benchmarking results by [60]. Two core challenges and limitations explain the slower convergence and reduced hypervolume performance of the intertemporal solution strategy relative to DPS: temporal salience structure and open loop control. The temporal salience structure of the lake problem refers to the differential effects of $\mathrm{P}$ release decisions over time [58], with earlier decisions having a greater influence on the economic benefits and reliability of the lake management plans 
due to the lake's irreversible dynamics and the discounting of economic benefits. Since the intertemporal solution strategy attempts to optimize 100 decision variables representing the $\mathrm{P}$ release decisions in each control period, the temporal salience structure of the lake problem results in sequential convergence of the release decisions from early to late stages, termed domino convergence [58]. Consequently, the least important decisions at the end of time do not strongly influence the hypervolume progress of the Borg MOEA, making it difficult for the algorithm's selection operators to optimize the $\mathrm{P}$ releases for these time steps. This results in slow convergence of the late-term decisions, termed drift stall [58].

The open loop control of the intertemporal strategy limits its final hypervolume performance due to its lack of feedback control compared to the closed loop DPS approach. Even though there are only 6 decision variables defining each DPS-derived policy function, when operated, the policy's feedback control enables different $\mathrm{P}$ release decisions in each realization of 100 years of random natural $\mathrm{P}$ inflows, as the release decisions respond to the different resulting trajectories of lake $\mathrm{P}$ concentrations. The open loop intertemporal strategy, however, finds only one set of 100 release decisions that must perform well on average across the 100 realizations of natural $\mathrm{P}$ inflows.

\subsection{Performance Comparison of the Different Control Strategies}

To analyze the control policies derived from the intertemporal and DPS solution strategies, we highlight a few solutions for further investigation. For each method, we select two solutions: that maximizing reliability and that maximizing economic benefits. Figure 4(b) shows the locations of these solutions in the Pareto-approximate sets and Table 2 summarizes their objective values. 
Table 3: Objective values of selected solutions from the intertemporal and DPS solution strategies. Low values are preferred for $\mathrm{O}_{2}$, while high values are preferred for the other three.

\begin{tabular}{cccccc}
\hline \hline \multirow{2}{*}{ Method } & Solution & $\begin{array}{c}\mathrm{O}_{1} \text { : Expected } \\
\text { Economic } \\
\text { Benefits }\end{array}$ & $\begin{array}{c}\mathrm{O}_{2} \text { : Maximum } \\
\text { Average P } \\
\text { Concentration }\end{array}$ & $\begin{array}{c}\mathrm{O}_{3}: \\
\text { Average } \\
\text { Inertia }\end{array}$ & $\begin{array}{c}O_{4}: \\
\text { Average } \\
\text { Reliability }\end{array}$ \\
\hline \hline \multirow{2}{*}{ Intertemporal } & $\begin{array}{c}\text { Highest } \\
\text { Reliability }\end{array}$ & 0.32 & 0.21 & 0.99 & 1.0 \\
\cline { 2 - 6 } & $\begin{array}{c}\text { Highest } \\
\text { Benefits }\end{array}$ & 0.43 & 2.25 & 0.97 & 0.86 \\
\hline \multirow{2}{*}{ DPS } & $\begin{array}{c}\text { Highest } \\
\text { Reliability }\end{array}$ & 0.34 & 0.22 & 0.99 & 1.0 \\
\cline { 2 - 6 } & Highest & 0.57 & 0.57 & 0.98 & 0.85 \\
\hline
\end{tabular}

\subsubsection{Policies vs. Intertemporal Pathways}

Figure 6(a) shows the shapes of the policies corresponding to the two DPS solutions, with the most reliable solution in blue and the benefits-maximizing solution in green. Also shown in red is the critical $\mathrm{P}$ threshold beyond which the lake is irreversibly eutrophic. As one would expect, both solutions map low $\mathrm{P}$ concentrations, $X_{t}$, to high $\mathrm{P}$ releases, $a_{t}$, and prescribe decreased releases as the lake $\mathrm{P}$ concentration increases. The most reliable policy is much more conservative, decreasing $\mathrm{P}$ discharges at much lower lake $\mathrm{P}$ concentrations than the benefits-maximizing solution. Both policies subsequently increase P discharges beyond some lake $\mathrm{P}$ concentration, with the benefits-maximizing solution doing so at much lower values. This is because tipping becomes inevitable as the lake $\mathrm{P}$ concentration approaches the critical threshold, and once the threshold is crossed, reliability can no longer be improved, so it is best to further maximize economic benefits. As is shown later, though, these policies are able to find oligotrophic equilibria below the critical $\mathrm{P}$ threshold such that the increasing region of the policy is rarely operated, especially under the most reliable policy. 


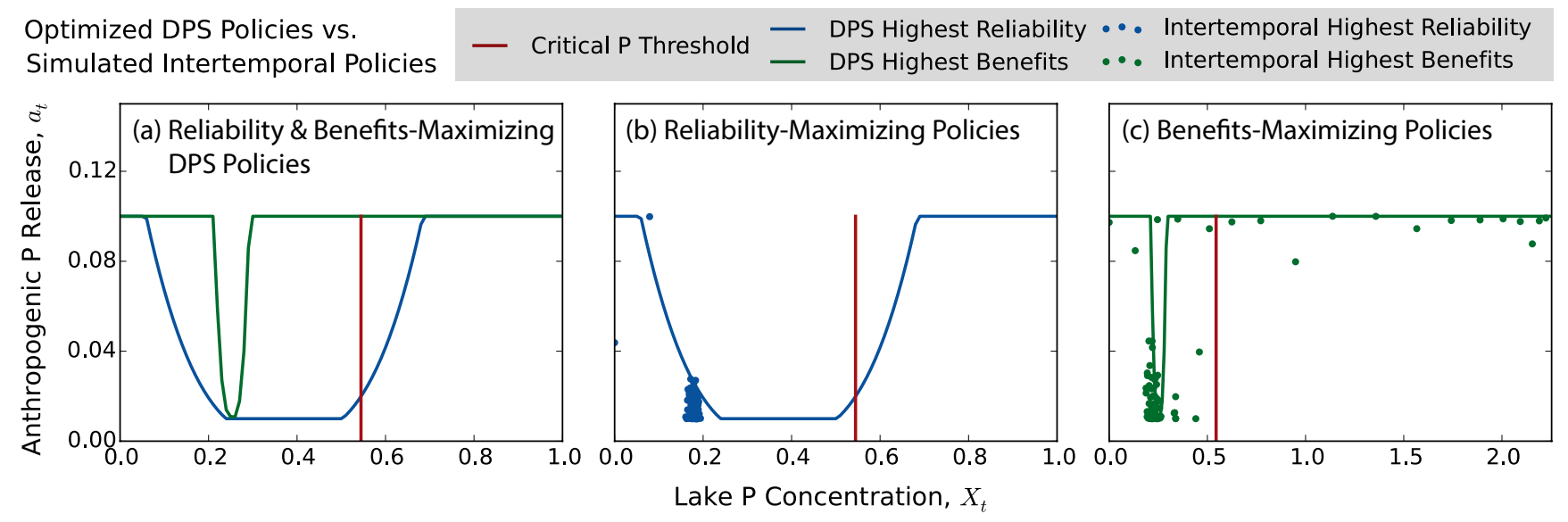

Figure 6: Optimized DPS policies vs. simulated intertemporal policies, where $\mathrm{P}$ release is plotted against the lake $\mathrm{P}$ concentration. Panel (a) compares the reliability-maximizing (blue) and benefits-maximizing (green) DPS policies, panel (b) compares the reliability-maximizing solutions from DPS (solid line) and the intertemporal solution strategy (points), and panel (c) compares the benefits-maximizing solutions from the two methods. The DPS policies are obtained directly from optimization, while proxy policies are obtained for the intertemporal policies by simulating them over their time horizon to determine the lake $\mathrm{P}$ concentration at the time of each $\mathrm{P}$ release. Consequently, the intertemporal policies are not continuous.

Figures 6(b) and (c) compare the two selected DPS policies with simulations of the corresponding intertemporal control strategies. Given that the intertemporal strategies are optimized release decisions at specific times, the time series of releases must be simulated to infer the corresponding lake $\mathrm{P}$ concentration when each release is made. For this reason, the decisions do not form a continuous function and have been plotted as points. As can be seen in Figure 6(b), the most reliable intertemporal policy only observes a small range of lake $\mathrm{P}$ concentrations since it is able to find an oligotrophic equilibrium about which the concentration fluctuates. If a manager wants to know how to operate this 
solution beyond the simulated horizon, though, the simulated policy is not a helpful decision tool due to its limited domain of applicability and the somewhat random corresponding decisions within that domain. The benefits-maximizing policy forms a more complete policy, although the randomness of this solution's decisions is also evident. The randomness in the intertemporal release decisions of both policies is largely driven by the large number of decision variables in the optimization, and the low sensitivity to later term decisions resulting in algorithmic drift stall [58], as discussed in Section 5.1.

\subsubsection{Time Series Comparison}

In addition to viewing the selected solutions in the policy space, it is informative to compare the time series of actual release decisions and corresponding lake $\mathrm{P}$ concentrations brought about by operating these policies. These time series are shown in Figure 7. As in Figure 6, the most reliable solutions are shown in blue, the benefits-maximizing in green, and the critical $\mathrm{P}$ threshold in red. The DPS solutions are represented by solid lines and the intertemporal solutions by dashed lines.

In the first row of this figure, the lake $\mathrm{P}$ concentration is plotted vs. time and one can see that all of the selected solutions quickly find a steady state $\mathrm{P}$ concentration at which the lake remains for most of the simulation. In the second row of Figure 7, where the $\mathrm{P}$ releases are plotted versus time, it becomes clear that these steady state $\mathrm{P}$ concentrations are quickly achieved by first releasing the maximum allowable $\mathrm{P}$ emission and then settling around a stable mean release. This is the same behavior [11] find to be optimal when managing the lake to maximize an economic utility function which penalizes crossing the critical $\mathrm{P}$ threshold. This strategy is also called "bang-bang" control [4], referring to the abrupt switch in control decisions from one extreme to another. In this case, bang-bang control is achieved by first releasing as much as possible in the first time step to reach a target steady state $\mathrm{P}$ concentration, and then drastically reducing emissions to a constant, lesser amount in all subsequent time steps to maintain equilibrium. 
While both the bang-bang strategy and the DPS solutions exhibit this behavior, the [11] optimization method only generates one solution that equilibrates to one $\mathrm{P}$ level found to be optimal according to a single utility function. Our multi-objective approach finds several solutions which equilibrate to different $\mathrm{P}$ levels depending on one's preference. In Figure 7(a), it can be seen that the benefits-maximizing DPS solution equilibrates to a slightly higher steady state $\mathrm{P}$ concentration than the most reliable DPS solution. The closer proximity of this steady state $\mathrm{P}$ concentration to the critical $\mathrm{P}$ threshold demonstrates the greater implicit risk of this policy.

Figures 7(b) and (c) compare the reliability and benefits-maximizing solutions found by each solution strategy, respectively. In panel 7(b), one can see that the selected solutions from both methods result in similar lake dynamics, with the selected intertemporal solution being slightly more conservative than the selected DPS solution, as it has a lower steady state P concentration. In panel 7 (c), however, the selected solutions from each method result in very different lake dynamics. Operation of the benefits-maximizing solution from the intertemporal optimization initially results in a fairly stable lake $\mathrm{P}$ concentration. However, after almost 80 years, the lake $\mathrm{P}$ concentration escapes this oligotrophic equilibrium and eventually crosses the critical $\mathrm{P}$ threshold, entering a eutrophic state. 

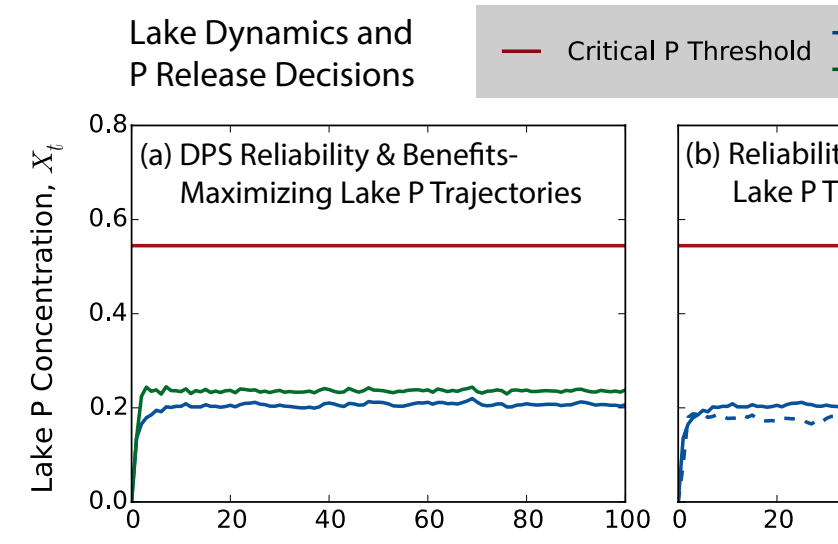

DPS Highest Reliability - - Intertemporal Highest Reliability

DPS Highest Benefits - - Intertemporal Highest Benefits
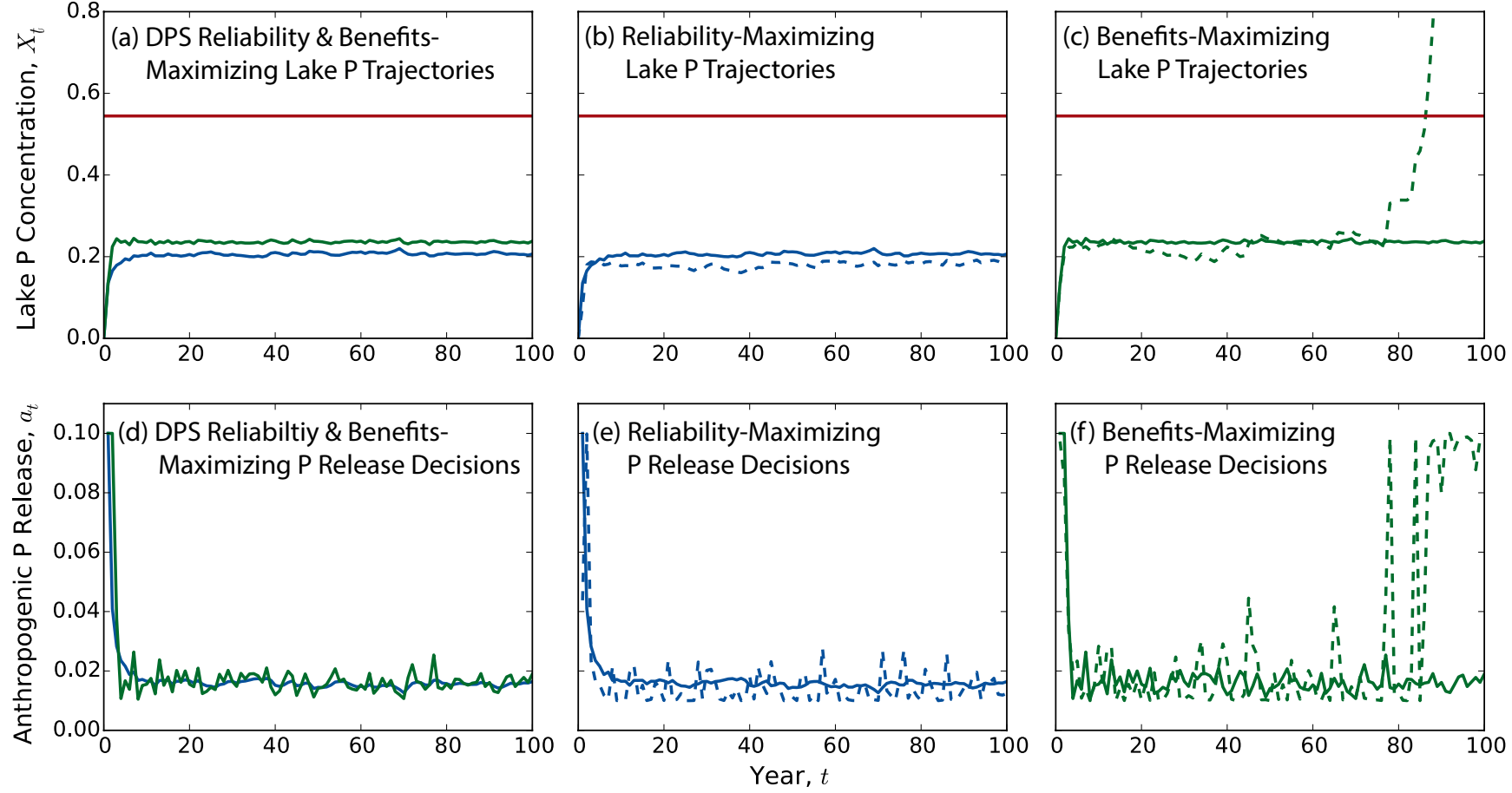

Figure 7: Time series of lake $\mathrm{P}$ concentration and anthropogenic $\mathrm{P}$ releases for select DPS and intertemporal solutions under one simulation of random natural $\mathrm{P}$ inflows. Panels (a) $-(\mathrm{c})$ show that all solutions quickly reach a stable oligotrophic equilibrium, but that the benefits-maximizing intertemporal policy crosses the critical P threshold near the end of the simulation, moving toward a eutrophic equilibrium. Panels (d)-(f) indicate that DPS release decisions, shown by solid lines, are more temporally stable than their intertemporal counterparts, shown by dashed lines. They also do not exhibit myopic end-of-time behavior, like the benefits-maximizing intertemporal solution. Additionally, the most reliable DPS solution is more stable than the benefits-maximizing DPS solution.

The decisions resulting in this behavior are shown in panel $7(\mathrm{f})$. Here one 
can see that after almost 80 years, the $\mathrm{P}$ release decisions from the benefitsmaximizing intertemporal solution suddenly increase dramatically. This solution prescribes conservative behavior initially to ensure that the lake $\mathrm{P}$ concentration stays below the critical $\mathrm{P}$ threshold for 85 years, meeting the reliability constraint. It then prescribes myopic behavior to maximize benefits, as if the end of the simulation were the end of time.

It should be noted that while these $\mathrm{P}$ releases at the end of time are much greater for the intertemporal solution than for the DPS solution shown in the same panel, the average economic benefits across 100 simulations of 100 years are actually greater for the DPS solution than the intertemporal solution (see Table 2). There are two reasons for this. The first is because the economic benefits are discounted, such that earlier $\mathrm{P}$ releases are much more important to the overall economic benefits than later $\mathrm{P}$ releases. The DPS solution is able to achieve greater average emissions early on without crossing the critical P threshold. The second reason is because the simulation shown in panel 7 (c) is from one realization of random natural $\mathrm{P}$ inflows. In other realizations, there are large enough natural $\mathrm{P}$ inflows to increase the lake $\mathrm{P}$ concentration to the rising limb of the benefits-maximizing DPS policy. In these realizations, perfect reliability is not achieved (note this solution's average reliability of $85 \%$ in Table 2) and economic benefits are greater than in the plotted realization.

While there are realizations in which $\mathrm{P}$ emissions from the benefits-maximizing DPS solution will bring the lake over the critical $\mathrm{P}$ threshold, the reason it does not always exhibit the end-of-time behavior observed by the benefits-maximizing intertemporal solution is because the $\mathrm{P}$ release decisions from the policy are conditioned solely on the current state of the lake, not on time. One could add time as an input to the DPS policy, and the objective values could improve from this additional information, but doing so would result in similar end-of-time behavior. This stresses the importance of carefully considering what one includes in the DPS policy, or how one formulates objectives.

In addition to preventing this undesirable behavior by conditioning the release decision solely on the state, the releases from the DPS solutions are also 
more stable in time than the releases from the intertemporal solutions, as seen in panels $7(\mathrm{e})$ and $7(\mathrm{f})$. This is again due to the difficulty for the MOEA to optimize later-term release decisions. It is also important to note from panel $7(\mathrm{~d})$ that the most reliable DPS policy is much more stable than the benefitsmaximizing DPS solution. This is because the benefits-maximizing DPS policy is much steeper about its observed oligotrophic equilibrium of just over 0.2 , as seen in Figure 6. A small change in the lake $\mathrm{P}$ concentration about this point demands a much greater change in the $\mathrm{P}$ release decision compared to the most reliable DPS policy. This is an additional, unforeseen tradeoff between economic benefits and reliability; in order to maximize economic benefits, one must also make a sacrifice in terms of temporal release stability.

\subsection{Robustness Analysis}

Sections 5.1 and 5.2 illustrate the computational and performance benefits of using DPS for many objective optimization of control problems, but one potential concern is that the optimal policies are too specific to the system to which they were optimized. If the true system characteristics are different, the solutions may no longer perform well. Using the MORDM framework [28] described in Section 4.2, we re-evaluate the performance of the solutions found by each solution strategy on alternative plausible lake systems to determine how robust they are to commonly deep uncertainties in characterizing environmental thresholds and economic discounting.

\subsubsection{Evaluation of Alternatives and Calculation of Robustness}

As explained in Section 4.2, we re-evaluate the Pareto-approximate solutions in 1,000 alternative SOWs and, for each solution, calculate robustness as the percent of SOWs in which three criteria are met: 1) economic benefits $>0.2,2$ ) average reliability $>95 \%$ and 3 ) both of these. In Figure 8, we have sorted the Pareto-approximate solutions found by each method by robustness under each of these three criteria, plotting the satisficing metric for the most robust solution on the left and the least robust on the right. Figure 9 shows the reference sets 
from the original optimization, with each point shaded by its robustness on the third criterion combining economic and reliability performance thresholds.

From Figure 8, it is clear that for all criteria, the DPS solutions in blue are more robust than the intertemporal solutions in red. However, robustness drops dramatically from one criterion to the next. Even the most robust DPS solution on criterion 3 satisfies the economic and reliability thresholds in less than $60 \%$ of sampled SOWs. Decision makers managing the lake would likely be concerned by this result. 


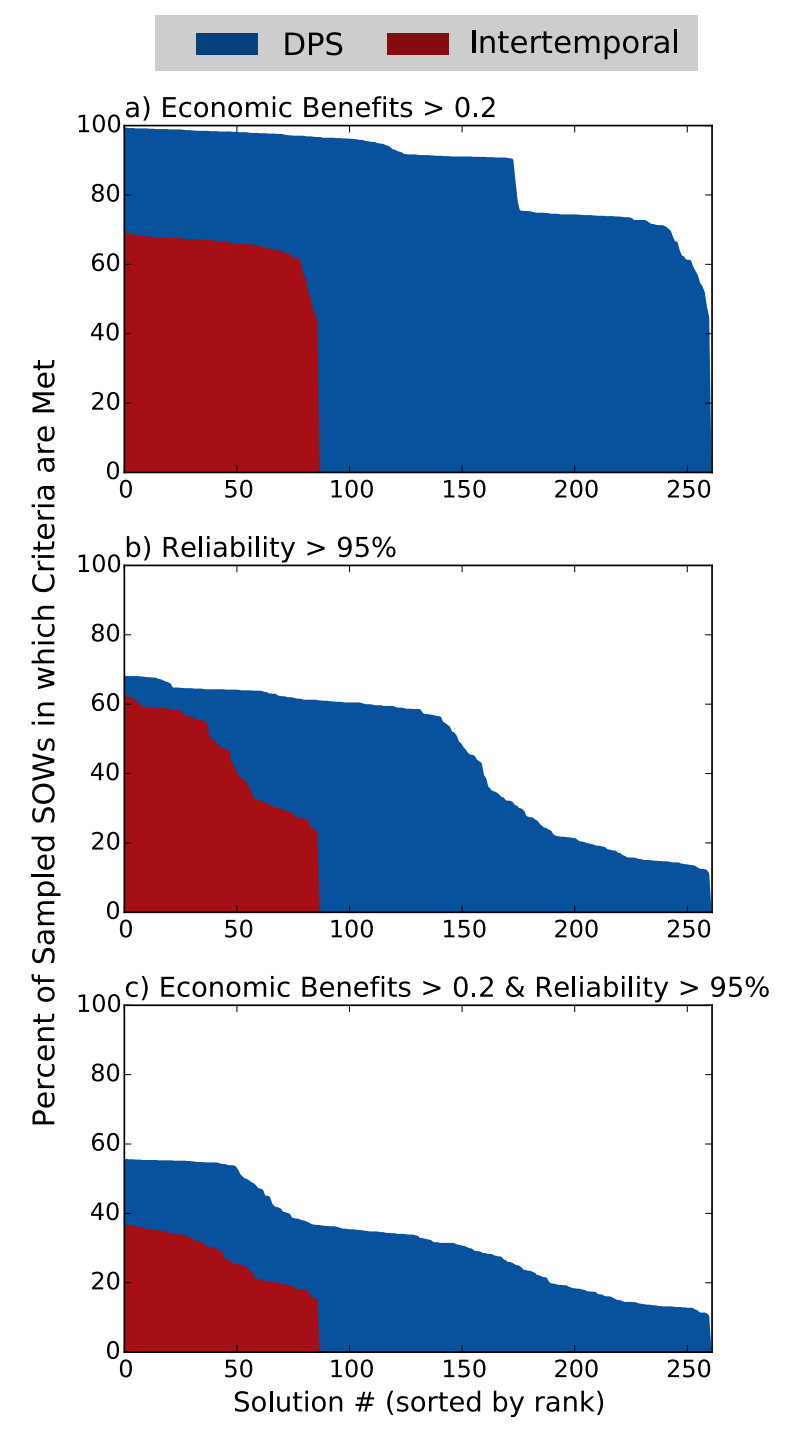

Figure 8: Robustness of DPS and intertemporal solutions according to the domain-satisficing criterion. Solutions from the reference sets of the DPS and intertemporal solution strategies are re-evaluated on 1,000 alternative SOWs and sorted by robustness. Robustness is quantified here as the percent of SOWs in which certain criteria are met: (a) economic benefits $>0.2$, (b) reliability $>$ $95 \%$ and (c) economic benefits $>0.2$ and reliability $>95 \%$. The DPS solutions are able to meet each of these criteria in a greater percent of the SOWs than the intertemporal solutions. 
To determine which parameter combinations are preventing these solutions from meeting criterion 3, we further analyze the most robust solutions from each solution strategy. These points are enlarged in Figure 9. Note the complex mapping between objective values and robustness of the DPS solutions that would make it difficult to predict the most robust solutions a priori, strongly limiting the value of classical multi-criterion decision making methods [12]. The DPS and intertemporal solutions with low economic benefits in the world to which they were optimized generally perform poorly on the robustness measure, likely failing to meet the economic criterion. Similarly, the intertemporal solutions with high maximum average $\mathrm{P}$ concentrations are not robust, likely failing to meet the reliability criterion. However, because many of the high benefits DPS solutions are able to achieve these benefits with low maximum average $\mathrm{P}$ concentrations, there is not an obvious failure mechanism on the reliability criterion.

This complex mapping of objective values to robustness highlights the importance of performing multi-objective search initially in order to find a diverse set of policy options, as it is often impossible to know a priori whether a singleobjective optimization method will find a robust solution. Since a solution's robustness may influence a decision maker's policy preference, it is better to perform multi-objective optimization to find a range of Pareto optimal policies and then re-evaluate them on alternative SOWs to determine how robust each solution is. Only once these robust solutions have been discovered can one determine which parameter uncertainties are driving its failures. 

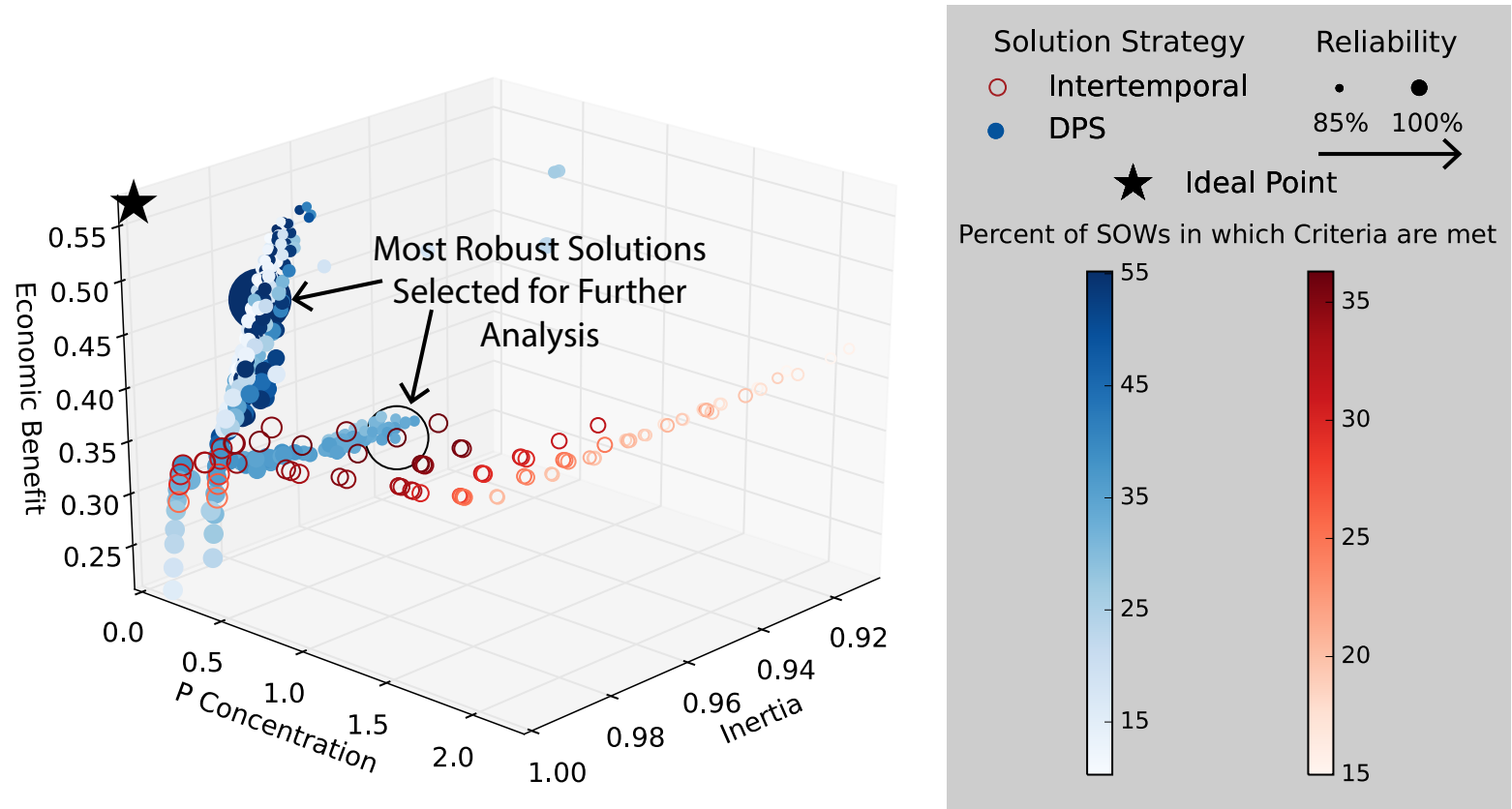

Figure 9: DPS and intertemporal reference sets shaded by robustness, with the most robust solutions enlarged. Robustness is defined as the percent of SOWs in which solutions have economic benefits $>0.2$ and reliability $>95 \%$. The complex spatial mapping to robustness, particularly of the DPS solutions, highlights the importance of a posteriori decision making; it would be impossible to predict in advance what weighted combination of objectives would yield robust solutions. The most robust solutions from each method, enlarged in the figure, are selected for further sensitivity analysis.

\subsubsection{Sensitivity Analysis of Uncertain Parameters}

In Figure 10, we have plotted 2-D projections of the sampled SOWs, with each point representing one of the 1,000 samples. The y-axis gives the value of $q$ in each point's SOW, while the x-axis gives the value of $b$ for the plots in the first column, and $\delta$ for the plots in the second. In the top row, each of these points has been colored green if the most robust DPS solution meets criterion 3 in that sampled SOW, and gray if it does not. In the bottom row, the same 
is done for the most robust intertemporal solution.

\section{Parameter Combinations Leading to Failure}
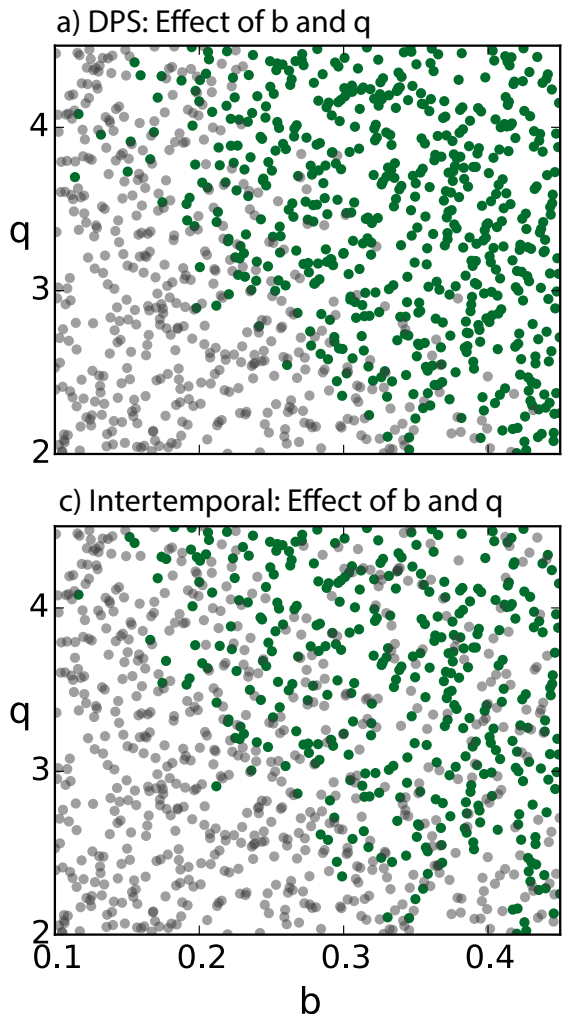

-. Meets Criteria

-.. Fails to Meet Criteria

b) DPS: Effect of $\delta$

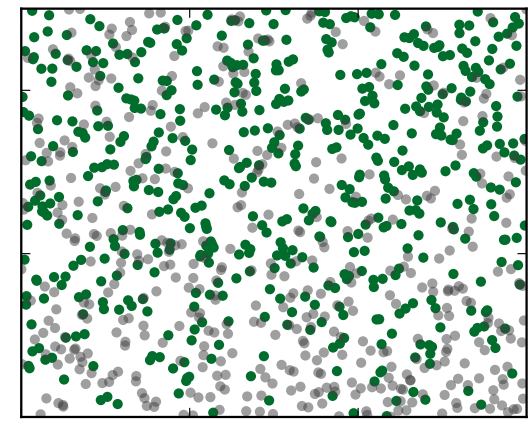

d) Intertemporal: Effect of $\delta$

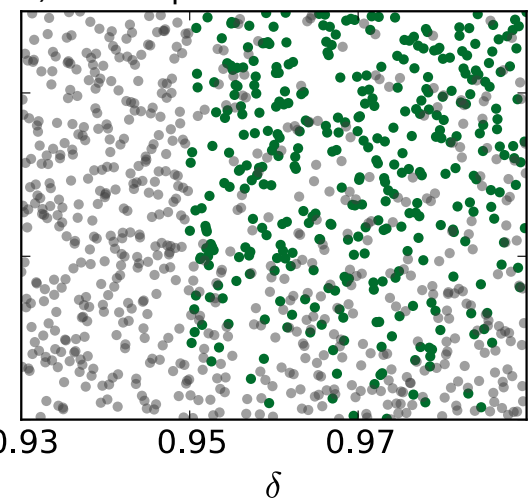

Figure 10: Combinations of uncertain parameter values leading to failure. Each dot represents a different SOW for a selected solution, with the most robust DPS solution shown in panels (a) and (b), and the most robust intertemporal solution in panels (c) and (d). Each dot is shaded green if the solution has economic benefits $>0.2$ and reliability $>95 \%$ in that SOW, or gray if it does not. Panels (a) and (c) show that there is a nonlinear combination of small values of $b$ and $q$ which always result in failure. Panels (b) and (d) show that there is a threshold value of the discount parameter, $\delta$, below which the most robust intertemporal solution always fails but the most robust DPS solution does not. 
In the first column, one can see that both the selected intertemporal and DPS solutions fail for a non-linear combination of low $b$ and low $q$ values. In the second column, however, differences emerge. The DPS solution is able to achieve criterion 3 for any of the sampled values of the discount parameter, $\delta$, used to calculate economic benefits. For the intertemporal policy, however, the selected solution is unable to achieve the economic threshold of criterion 3 for values of $\delta$ below about 0.95 . Because the intertemporal solution prescribes the same time series of $\mathrm{P}$ release decisions in every realization, regardless of the other lake parameters, its economic performance is sensitive only to the discount parameter. While the economic benefits of the DPS solution also depend on this parameter, the $\mathrm{P}$ release decisions from this policy change depending on the state of the lake, and the lake $\mathrm{P}$ concentration follows a different trajectory under different system parameters. By adapting its $\mathrm{P}$ release decisions to the state of the lake, the selected DPS solution becomes insensitive to the discount parameter and is able to reach high economic benefits regardless of that parameter's value.

\subsubsection{Effect of the critical $P$ threshold on policy failure}

While the insensitivity of the most robust DPS solution to the discount parameter was an unforeseen benefit of the method, the remaining sensitivity to $b$ and $q$ is concerning. Figure 11 shows that the non-linear combination of $b$ and $q$ causing these failures is actually driven primarily by the critical $\mathrm{P}$ threshold associated with these parameter combinations. This figure shows a contour map of the critical P threshold laid atop the dot plot in Figure 10(a), but with successes now plotted black instead of green. The critical P threshold below which the policies almost always fail lies around the 0.5 contour. The critical P threshold in the world to which the DPS policies were optimized is 0.54 , indicating a high sensitivity of the policies to this threshold. Just a small movement to a lower threshold results in it being crossed, likely due to the inexistence of an oligotrophic equilibrium when the policy is operated on a lake system with this threshold. 


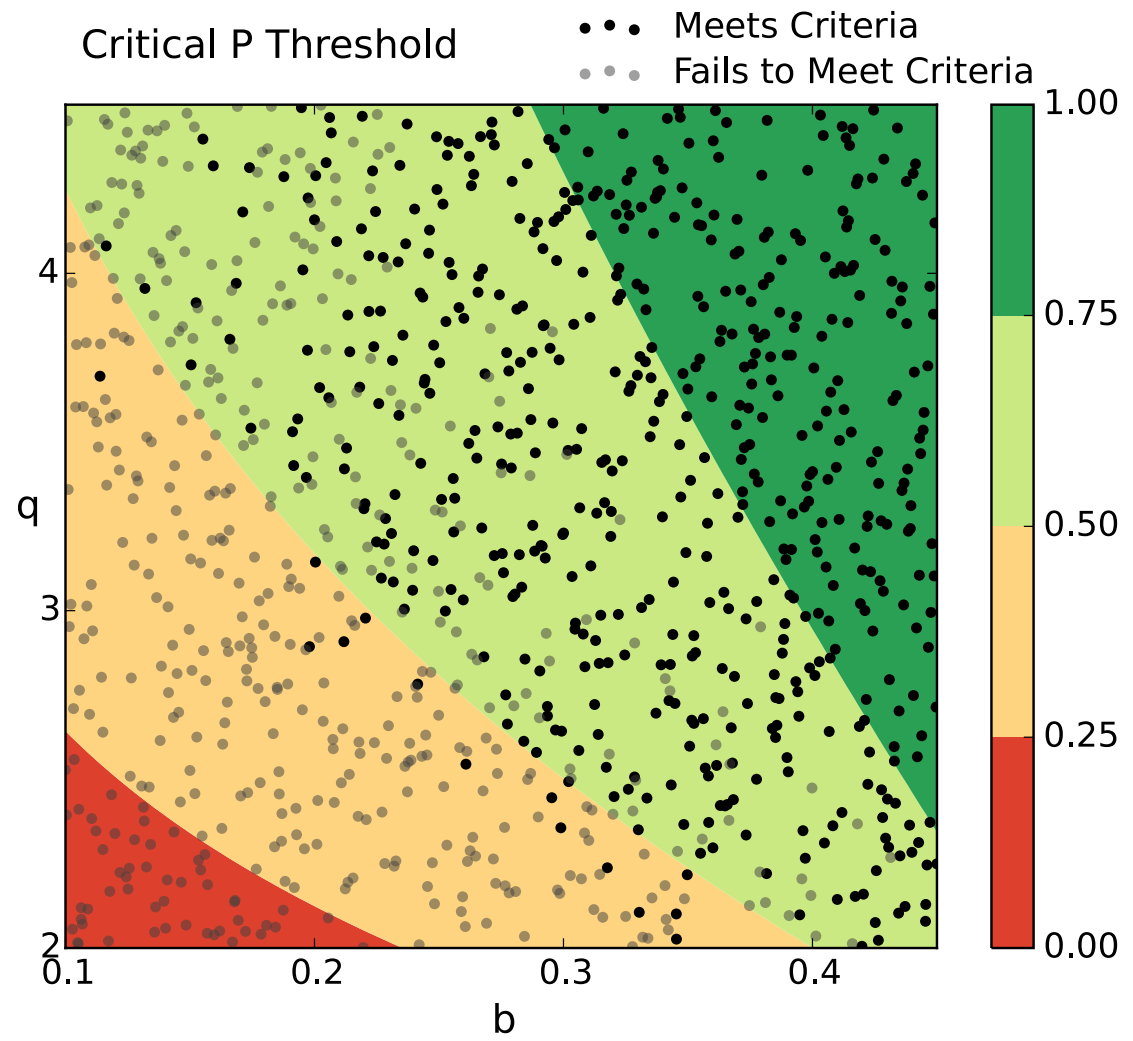

Figure 11: Effect of critical $P$ threshold on policy failure. This is the same dot plot as shown in Figure 10(b), but atop a contour plot of the critical P threshold as a function of $b$ and $q$. One can see that below a critical $\mathrm{P}$ threshold of about 0.5 , the most robust DPS solution always fails to meet the satisficing criteria. This is very close to the critical threshold of 0.54 in the SOW to which the policy was optimized, indicating high sensitivity to the assumed model parameters.

Furthermore, there is a littering of failures even just above this threshold, with the failure rate increasing for higher values of $q$. In this region, the impact of the other uncertain parameters becomes important in determining whether or not the policy will succeed. For example, high values of $q$ result in more abrupt transitions across the threshold due to steeper recycling curves, while greater values of $\mu$ result in natural $\mathrm{P}$ inputs eliminating the system's oligotrophic 
equilibrium.

Clearly, one approach to improve robustness is to improve the understanding of the uncertain parameters and, in response, adjust the policy so that it maintains an oligotrophic equilibrium. Fortunately, the ability of DPS to incorporate additional information into the policy could enable the formation of more adaptive policies. For example, the DPS policy could include the mean and variance of one's estimate of the critical $\mathrm{P}$ threshold, and change adaptively as these estimates are refined through endogenous learning [36, 29]. Alternatively, one could directly include estimates of the critical $\mathrm{P}$ threshold rather than both $b$ and $q$. Prior work on tipping points has found that systems tend to slow down as they approach tipping points [52], so the rate of change in lake $\mathrm{P}$ concentration could potentially be a DPS indicator as well. We are pursuing these avenues in further research.

\section{Conclusions}

Managing socio-ecological systems for multiple objectives poses several challenges, particularly if the systems possess non-linear tipping points and deeply uncertain system dynamics. In this study, we use the classical shallow lake problem to illustrate how DPS can alleviate many of the difficulties associated with identifying robust environmental control policies for these systems, as well as their inherent tradeoffs. By directly parameterizing operating policies for the system and optimizing the parameters of those policies with an MOEA, DPS can exploit the information available in detailed system simulations. Our results show that the DPS-based closed loop control strategy can drastically reduce the computational complexities of solving a benchmark many-objective control problem compared to optimizing open loop control policies. Additionally, the control feedback inherent to DPS policies results in more flexible system operations that can react to different observations of stochastic inputs. While the actual use of an intertemporal open loop control policy without re-optimization requires that one take a particular action regardless of the resulting system state, 
operation of a closed loop control policy found with DPS enables different decisions based on the system's state trajectory without having to re-optimize the policies at every time step. This adaptability makes DPS policies more robust to well-characterized uncertainty in stochastic inputs such as natural P loads to a lake, enabling better performance across a stochastic ensemble of potential inputs.

The adaptability of DPS policies not only yields improved performance under well-characterized uncertainty in stochastic inputs, but also improved robustness to deep uncertainties. In this study, we find that DPS policies optimized to an assumed SOW generalize better when subjected to alternative SOWs than intertemporal control policies. In particular, the DPS policies are able to meet minimum environmental and economic performance criteria in a larger fraction of alternative SOWs than the intertemporal policies. In fact, the most robust DPS policy is able to adapt its decisions well enough in alternative SOWs that its ability to meet the criteria is insensitive to the value of the discount parameter used to calculate economic benefits, which is not true of the most robust intertemporal policy.

Finally, the ability of DPS to include multiple sources of information into the optimized control policies allows one to create even more flexible policies that include estimates of the system parameters and their associated uncertainty. This information could prevent policies optimized to an assumed SOW from crossing a catastrophic tipping point below what was assumed, or conversely from behaving too conservatively in systems with tipping points above what was assumed. DPS could therefore be a simple adaptive management tool for identifying robust control policies in socio-ecological systems like the lake problem that exhibit deeply uncertain threshold behavior. The most obvious extension is to the Earth system and its many components that could be on the brink of a tipping point induced by anthropogenic climate change. Future work in this area should focus on developing state-dependent rather than intertemporal $\mathrm{CO}_{2}$ emissions policies and adaptively changing these policies as one gains new information on system conditions critical to tipping. 


\section{Acknowledgements}

We thank G. Garner, D. Hadka, J. Herman, J. Lamontagne, P. Oddo and J. Stedinger for valuable inputs. This work was partially supported by the National Science Foundation (NSF) through the Network for Sustainable Climate Risk Management (SCRiM) under NSF cooperative agreement GEO-1240507 and the Penn State Center for Climate Risk Management. Any opinions, findings, and conclusions or recommendations expressed in this material are those of the author(s) and do not necessarily reflect the views of the funding entities.

\section{References}

[1] Admiraal, J. F., Wossink, A., de Groot, W. T., \& de Snoo, G. R. (2013). More than total economic value: How to combine economic valuation of biodiversity with ecological resilience. Ecological Economics, 89, 115-122.

[2] Anderies, J. M., Carpenter, S. R., Steffen, W., \& Rockström, J. (2013). The topology of non-linear global carbon dynamics: From tipping points to planetary boundaries. Environmental Research Letters, 8, 044048.

[3] Bankes, S. (1993). Exploratory modeling for policy analysis. Operations research, 41, 435-449.

[4] Bellman, R., Glicksberg, I., \& Gross, O. (1955). On the'bang-bang'control problem. Technical Report DTIC Document.

[5] Bertsekas, D. P. (1995). Dynamic programming and optimal control volume 1. Athena Scientific Belmont, MA.

[6] Breiman, L., Friedman, J., Stone, C. J., \& Olshen, R. A. (1984). Classification and regression trees. CRC press.

[7] Brown, C., Ghile, Y., Laverty, M., \& Li, K. (2012). Decision scaling: Linking bottom-up vulnerability analysis with climate projections in the water sector. Water Resources Research, 48, W09537. 
[8] Bryant, B. P., \& Lempert, R. J. (2010). Thinking inside the box: A participatory, computer-assisted approach to scenario discovery. Technological Forecasting and Social Change, 77, 34-49.

[9] Buşoniu, L., Ernst, D., De Schutter, B., \& Babuška, R. (2011). Crossentropy optimization of control policies with adaptive basis functions. Systems, Man, and Cybernetics, Part B: Cybernetics, IEEE Transactions on, 41, 196-209.

[10] Carpenter, S. R., Brock, W. A., Folke, C., van Nes, E. H., \& Scheffer, M. (2015). Allowing variance may enlarge the safe operating space for exploited ecosystems. Proceedings of the National Academy of Sciences, 112, 14384-14389.

[11] Carpenter, S. R., Ludwig, D., \& Brock, W. A. (1999). Management of eutrophication for lakes subject to potentially irreversible change. Ecological applications, 9, 751-771.

[12] Chankong, V., \& Haimes, Y. (1983). Multiobjective Decision Making: Theory and Methodology. New York: North-Holland.

[13] Cohon, J., \& Marks, D. (1975). A review and evaluation of multiobjective programming techniques. Water Resources Research, 11, 208-220.

[14] Cui, L.-J., \& Kuczera, G. (2005). Optimizing urban water supply headworks operating rules under stochastic inputs: Assessment of genetic algorithm performance. Water Resources Research, 41. doi:10.1029/ 2004 WR003517.

[15] Dalal, S., Han, B., Lempert, R., Jaycocks, A., \& Hackbarth, A. (2013). Improving scenario discovery using orthogonal rotations. Environmental Modelling 83 Software, 48, 49-64. URL: http://www.sciencedirect.com/ science/article/pii/S1364815213001345. 
[16] Dessai, S., Hulme, M., Lempert, R., \& Pielke Jr, R. (2009). Climate prediction: a limit to adaptation. Adapting to climate change: thresholds, values, governance, (pp. 64-78).

[17] Friedman, J. H., \& Fisher, N. (1999). Bump hunting in high-dimensional data. Stat. Comput., 9, 123-143.

[18] Giuliani, M., Castelletti, A., Pianosi, F., Mason, E., \& Reed, P. M. (2016). Curses, tradeoffs, and scalable management: Advancing evolutionary multiobjective direct policy search to improve water reservoir operations. Journal of Water Resources Planning and Management, (p. 04015050).

[19] Guariso, G., Rinaldi, S., \& Soncini-Sessa, R. (1986). The management of lake como: A multiobjective analysis. Water Resources Research, 22, 109-120.

[20] Haasnoot, M., Kwakkel, J. H., Walker, W. E., \& ter Maat, J. (2013). Dynamic adaptive policy pathways: A method for crafting robust decisions for a deeply uncertain world. Global Environmental Change, 23, 485-498. URL: http://www. sciencedirect.com/science/article/pii/ S095937801200146X. doi:10.1016/j.gloenvcha.2012.12.006.

[21] Hadka, D., Herman, J., Reed, P., \& Keller, K. (2015). An open source framework for many-objective robust decision making. Environmental Modelling $\&$ Software, 74, 114-129.

[22] Hadka, D., \& Reed, P. (2013). Borg: An auto-adaptive many-objective evolutionary computing framework. Evolutionary Computation, 21, 231259.

[23] Hall, J., Lempert, R. J., Keller, K., Hackbarth, A., Mijere, C., \& McInerney, D. J. (2012). Robust climate policies under uncertainty: A comparison of robust decision making and info-gap methods. Risk Analysis, . doi:10.111 j .1539-6924.2012.01802.x. 
[24] Herman, J., Zeff, H., Reed, P., \& Characklis, G. (2014). Beyond optimality: Multi-stakeholder robustness tradeoffs for regional water portfolio planning under deep uncertainty. Water Resources Research, (p. [In Press]). doi:10 . 1002/2014WR015338.

[25] Herman, J. D., Reed, P. M., Zeff, H. B., \& Characklis, G. W. (2015). How should robustness be defined for water systems planning under change? Journal of Water Resources Planning and Management, 141, 04015012.

[26] Hine, D., \& Hall, J. W. (2010). Information gap analysis of flood model uncertainties and regional frequency analysis. Water Resources Research, 46. doi:10.1029/2008WR007620.

[27] Kasprzyk, J. R. (2013). Many Objective Water Resources Planning and Management Given Deep Uncertainties, Population Pressures, and Environmental Change. Ph.D. thesis The Pennsylvania State University.

[28] Kasprzyk, J. R., Nataraj, S., Reed, P. M., \& Lempert, R. J. (2013). Many objective robust decision making for complex environmental systems undergoing change. Environmental Modelling and Software, 42, 55-71. doi:10.1016/j.envsoft .2012.12.007.

[29] Keller, K., \& McInerney, D. (2008). The dynamics of learning about a climate threshold. Climate Dynamics, 30, 321-332. URL: http://link. springer.com/article/10.1007/s00382-007-0290-5.

[30] Keller, K., Yohe, G., \& Schlesinger, M. (2008). Managing the risks of climate thresholds: uncertainties and information needs. Climatic Change, 91, 5-10. URL: http://www.springerlink.com/index/ E550754338771K25.pdf.

[31] Knight, F. H. (1921). Risk, Uncertainty, and Profit. Boston, MA: Houghton Mifflin. 
[32] Koutsoyiannis, D., \& Economou, A. (2003). Evaluation of the parameterization-simulation-optimization approach for the control of reservoir systems. Water Resources Research, 39.

[33] Kwakkel, J. H., Walker, W. E., \& Haasnoot, M. (2016). Coping with the wickedness of public policy problems: Approaches for decision making under deep uncertainty. Journal of Water Resources Planning and Management, (p. 01816001).

[34] Laumanns, M., Thiele, L., Deb, K., \& Zitzler, E. (2002). Combining convergence and diversity in evolutionary multiobjective optimization. Evolutionary computation, 10, 263-282.

[35] Lempert, R., Popper, S., \& Bankes, S. (2002). Confronting surprise. Social Science Computer Review, 20, 420-440.

[36] Lempert, R. J., \& Collins, M. (2007). Managing the risk of an uncertain threshold response: Comparison of robust, optimimum, and precautionary approaches. Risk Analysis, 27, 1009-1026.

[37] Lempert, R. J., \& Groves, D. G. (2010). Identifying and evaluating robust adaptive policy responses to climate change for water management agencies in the American west. Technological Forecasting and Social Change, 77, 960-974.

[38] Lempert, R. J., Groves, D. G., Popper, S. W., \& Bankes, S. C. (2006). A general, analytic method for generating robust strategies and narrative scenarios. Management Science, 52, 514-528.

[39] Lempert, R. J., Popper, S. W., \& Bankes, S. C. (2010). Robust decision making: coping with uncertainty. The Futurist, 44, 47.

[40] Lenton, T. M. (2013). Environmental tipping points. Annual Review of Environment and Resources, 38, 1-29. 
[41] Lenton, T. M., Held, H., Kriegler, E., Hall, J. W., Lucht, W., Rahmstorf, S., \& Schellnhuber, H. J. (2008). Tipping elements in the earth's climate system. Proceedings of the national Academy of Sciences, 105, 1786-1793.

[42] Moody, P., \& Brown, C. (2012). Modeling stakeholder-defined climate risk on the upper great lakes. Water Resources Research, 48 .

[43] Nicklow, J., Reed, P., Savic, D., Dessalegne, T., Harrell, L., Chan-Hilton, A., Karamouz, M., Minsker, B., Ostfeld, A., Singh, A., \& Zechman, E. (2010). State of the art for genetic algorithms and beyond in water resources planning and management. Journal of Water Resources Planning and Management, 136, 412-432.

[44] Nordhaus, W. D. (2013). The climate casino: Risk, uncertainty, and economics for a warming world. Yale University Press.

[45] Pareto, V. (1896). Cours D'Economie Politique. Lausanne: Rouge.

[46] Parry, M. L. (2007). Climate change 2007-impacts, adaptation and vulnerability: Working group II contribution to the fourth assessment report of the IPCC volume 4. Cambridge University Press.

[47] Reed, P. M., Hadka, D., Herman, J. D., Kasprzyk, J. R., \& Kollat, J. B. (2013). Evolutionary multiobjective optimization in water resources: The past, present and future. Advances in Water Resources, 51, 438-456.

[48] Rosenstein, M. T., \& Barto, A. G. (2001). Robot weightlifting by direct policy search. In International Joint Conference on Artificial Intelligence (pp. 839-846). Citeseer volume 17.

[49] Salazar, J. Z., Reed, P. M., Herman, J. D., Giuliani, M., \& Castelletti, A. (2016). A diagnostic assessment of evolutionary algorithms for multiobjective surface water reservoir control. Advances in Water Resources, 92, $172-185$. 
[50] Saltelli, A. (2002). Making best use of model evaluations to compute sensitivity indices. Computer Physics Communications, 145, 280-297.

[51] Savage, L. J. (1951). The theory of statistical decision. Journal of the American Statistical association, 46, 55-67.

[52] Scheffer, M., Bascompte, J., Brock, W. A., Brovkin, V., Carpenter, S. R., Dakos, V., Held, H., Van Nes, E. H., Rietkerk, M., \& Sugihara, G. (2009). Early-warning signals for critical transitions. Nature, 461, 53-59.

[53] Scheffer, M., Hosper, S., Meijer, M., Moss, B., \& Jeppesen, E. (1993). Alternative equilibria in shallow lakes. Trends in ecology $\&$ evolution, 8 , 275-279.

[54] Singh, R., Reed, P. M., \& Keller, K. (2015). Many-objective robust decision making for managing an ecosystem with a deeply uncertain threshold response. Ecol. Soc, 20, 12.

[55] Sobol, I. (2001). Global sensitivity indices for nonlinear mathematical models and their Monte Carlo estimates. Mathematics and Computers in Simulation, 55, 271-280.

[56] Solomon, S., Plattner, G., Knutti, R., \& Friedlingstein, P. (2009). Irreversible climate change due to carbon dioxide emissions. Proceedings of the National Academy of Sciences, 106, $1704-1709$.

[57] Starr, M. K. (1962). Product Design and Decision Theory. Prentice-Hall, Englewood Cliffs, N.J.

[58] Thierens, D., Goldberg, D. E., \& Pereira, A. G. (1998). Domino convergence, drift, and the temporal-salience structure of problems. In The 1998 IEEE International Conference on Evolutionary Computation (pp. $535-540)$.

[59] Walker, W. E., Harremoës, P., Rotmans, J., van der Sluijs, J. P., van Asselt, M. B., Janssen, P., \& Krayer von Krauss, M. P. (2003). Defining 
uncertainty: a conceptual basis for uncertainty management in model-based decision support. Integrated assessment, 4, 5-17.

[60] Ward, V. L., Singh, R., Reed, P. M., \& Keller, K. (2015). Confronting tipping points: Can multi-objective evolutionary algorithms discover pollution control tradeoffs given environmental thresholds? Environmental Modelling 85 Software, 73, 27-43.

[61] Werners, S. E., Pfenninger, S., van Slobbe, E., Haasnoot, M., Kwakkel, J. H., \& Swart, R. J. (2013). Thresholds, tipping and turning points for sustainability under climate change. Current opinion in environmental sustainability, 5, 334-340.

[62] Zitzler, E., Thiele, L., Laumanns, M., Fonseca, C. M., \& Fonseca, V. G. d. (2003). Performance assessment of multiobjective optimizers: an analysis and review. IEEE Transactions on Evolutionary Computation, 7, 117-132. doi:10.1109/tevc.2003.810758. 
a) Open Loop Control

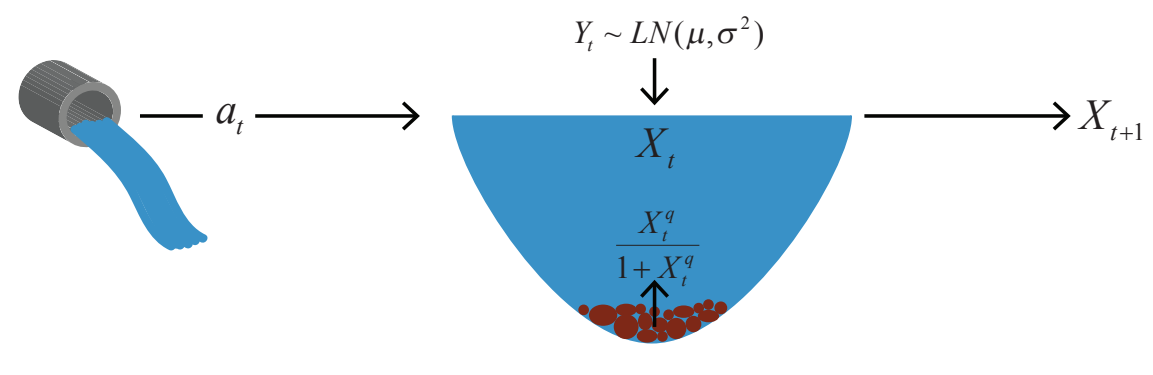

b) Closed Loop Control

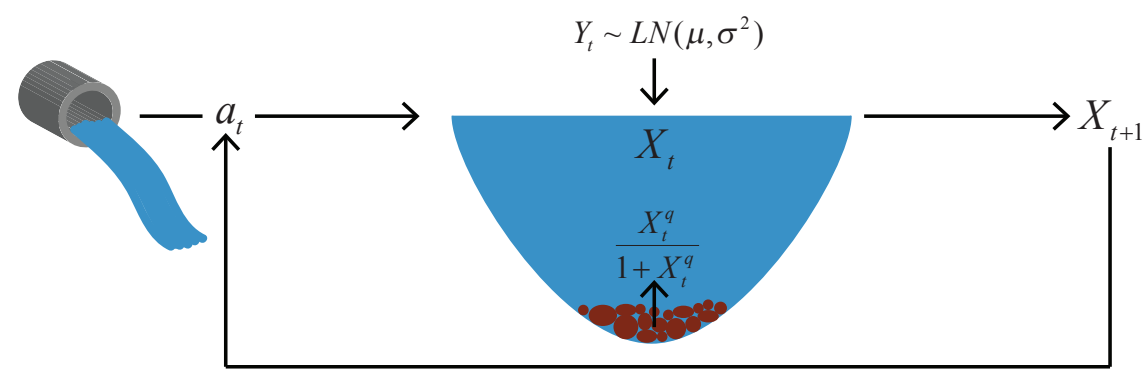

Figure A1: Open vs. closed loop control. In open loop control (panel a), the control decision, here the anthropogenic $\mathrm{P}$ release decision, $a_{t}$, is made without any feedback from the system state, here the $\mathrm{P}$ concentration in the lake, $X_{t}$. In closed loop control (panel b), the lake $\mathrm{P}$ concentration resulting from the control decision, $X_{t+1}$, feeds back into the control decision for the next time step, $a_{t+1}$. 